\title{
MODELLING AND SENSITIVITY ANALYSIS OF UNCOORDINATED PAIRED INTERSECTIONS WITH LEFT-TURN BAYS
}

\author{
Ronghan YAO*, Wensong ZHANG, Meng LONG \\ School of Transportation and Logistics, Dalian University of Technology, China \\ Received 12 June 2018; revised 31 August 2019; accepted 22 September 2019; \\ first published online 26 March 2020
}

\begin{abstract}
Left-turn bays are often installed on the road segment between paired intersections. Such left-turn bays may reduce the approach capacities and impact on one another. Four optimization models are put forward for uncoordinated paired intersections with left-turn bays. The phase effective green times and the left-turn bay lengths are the decision variables, maximizing the intersection capacities, minimizing the intersection delays and both of them are respectively regarded as different objective functions, and minimizing the total delay for paired intersections is viewed as another objective function. The total capacity-to-delay ratio is defined to evaluate the operations of paired intersections as a whole. Using the field data, the sensitivities of the optimized outcomes to the weighting factors of the objective functions are analysed. To clarify the influences of different scenarios on traffic stream operations, seven scenarios are tested using VISSIM. The interval estimation and hypothesis testing are used to analyse the simulated data. Three concrete models are recommended to apply in practice with the procedure of model application being provided. The achievements can be applied to optimally assign the temporal-spacial resources for paired intersections when left-turn bays need to be installed and coordinated signals do not need to be considered.
\end{abstract}

Keywords: paired intersections, left-turn bays, uncoordinated signals, signal timings, optimization, sensitivity analysis.

\section{Notations}

\section{Abbreviations:}

$$
\begin{aligned}
& \text { CP - existing scenario; } \\
& \text { WP - Webster's scenario; } \\
& \text { max - maximize; } \\
& \text { min - minimize; } \\
& \text { s.t. - constraints. }
\end{aligned}
$$

Variables and functions:

$Q_{j}^{\eta}, S_{f j}^{\eta}, S_{s j}^{\eta}$ - capacity, full-lane saturation flow rate, and left-turn bay saturation flow rate for lane group $j$ at intersection $\eta$, respectively $[\mathrm{pcu} / \mathrm{h}]$;

$\phi_{i j}^{\eta}$ - indicator for identifying whether vehicles in lane group $j$ can traverse the intersection in phase $i$ at intersection $\eta$, if yes, $\phi_{i j}^{\eta}=1$, otherwise $-\phi_{i j}^{\eta}=0$;

$\delta_{j}^{\eta}$ - indicator for identifying whether a left-turn bay is added in lane group $j$ at intersection $\eta$, if yes, $\delta_{j}^{\eta}=1$, otherwise $-\delta_{j}^{\eta}=0$; $n^{\eta}$ - number of phases for intersection $\eta$;

$g_{i}^{\eta}, g_{j}^{\eta}$ - effective green time for phase $i$ and left-turn bay queue full discharge time for lane group $j$ at intersection $\eta$, separately [s];

$C^{\eta}$ - cycle length for intersection $\eta[s]$;

$L^{\eta}$ - total lost time for intersection $\eta[s]$;

$D_{j}^{\eta}$ - left-turn bay length for lane group $j$ at intersection $\eta[\mathrm{m}]$;

$h, \bar{t}$ - average queue spacing [m] and saturation headway [s] between consecutive vehicles, respectively, and they are set to $6 \mathrm{~m}$ and $2 \mathrm{~s}$ in this paper;

$Q^{\eta}, m^{\eta}$ - capacity $[\mathrm{pcu} / \mathrm{h}]$ and number of lane groups for intersection $\eta$;

$d_{j}^{\eta}, x_{j}^{\eta}, q_{j}^{\eta}$ - average delay [s/pcu], saturation degree, and arrival flow rate $[\mathrm{pcu} / \mathrm{h}]$ for lane group $j$ at intersection $\eta$, respectively;

$u_{j}^{\eta}$ - green ratio for lane group $j$ at intersection $\eta$;

${ }^{\star}$ Corresponding author. E-mail: cyanyrh@dlut.edu.cn 
$T$ - analysis period [h], and a value of $1 \mathrm{~h}$ is adopted in this paper;

$d^{\eta}$ - average delay for intersection $\eta[\mathrm{s} / \mathrm{pcu}]$

$\tilde{g}_{\min i}^{\eta}, L_{p j}^{\eta}$ - pedestrian effective green time for phase $i$ [s] and crosswalk length for lane group $j[\mathrm{~m}]$ at intersection $\eta$, separately;

$\varphi_{j}^{\eta}$ - indicator for identifying whether vehicles in lane group $j$ can traverse intersection $\eta$ in a single phase, if yes, $\varphi_{j}^{\eta}=1$, otherwise $\varphi_{j}^{\eta}=0$;

$v_{p}-$ pedestrian walking speed $[\mathrm{m} / \mathrm{s}]$, and it is set to $1.2 \mathrm{~m} / \mathrm{s}$ in this paper;

$I_{i}^{\eta}$ - intergreen time between phase $i$ and the next phase at intersection $\eta[s]$;

$C_{\text {min }}^{\eta}, Y^{\eta}-$ minimal cycle length [s] and total flow ratio for intersection $\eta$, respectively;

$y_{j}^{\eta}$ - flow ratio for lane group $j$ at intersection $\eta$;

$C_{\max }^{\eta}-$ maximal cycle length for intersection $\eta[s]$; estimated minimal and maximal effective

$\hat{g}_{\min i}^{\eta}, g_{\max i}^{\eta}-$ green times for phase $i$ at intersection $\eta$, separately $[s]$;

$g_{\min i}^{\eta}$ - minimal effective green time for phase $i$ at intersection $\eta[s]$;

$D_{\max j}^{\eta}$ - maximal left-turn bay length for lane group $j$ at intersection $\eta[\mathrm{m}]$, and a value of $300 \mathrm{~m}$ is used in this paper;

$\gamma_{k}^{\mathrm{A}}$ - indicator for identifying whether the leftturn bay in lane group $k$ at intersection A is installed on the road segment between paired intersections, if yes, $\gamma_{k}^{\mathrm{A}}=1$, otherwise $-\gamma_{k}^{\mathrm{A}}=0$;

$\gamma_{l}^{\mathrm{B}}$ - indicator for identifying whether the leftturn bay in lane group $l$ at intersection B is installed on the road segment between paired intersections, if yes, $\gamma_{l}^{\mathrm{B}}=1$, otherwise $-\gamma_{l}^{\mathrm{B}}=0$;

$D_{k}^{\mathrm{A}}$ - left-turn bay length for lane group $k$ at intersection A [m];

$D_{l}^{\mathrm{B}}-$ left-turn bay length for lane group $l$ at intersection B [m];

$D_{0}$ - road segment length for paired intersections $[\mathrm{m}]$;

$T D$ - total delay for paired intersections $[\mathrm{s}]$;

$d^{A}, d^{B}$ - average delays for intersections $\mathrm{A}$ and $\mathrm{B}$, respectively $[\mathrm{s} / \mathrm{pcu}]$;

$q^{A}, q^{B}$ - arrival flow rates for intersections $\mathrm{A}$ and $\mathrm{B}$, separately $[\mathrm{pcu} / \mathrm{h}]$;

$m^{A}, m^{B}$ - number of lane groups for intersections A and $\mathrm{B}$, separately;

$q_{j}^{\mathrm{A}}, q_{j}^{\mathrm{B}}$ - arrival flow rates for lane group $j$ at intersections $A$ and $B$, respectively [pcu/h];

$d_{j}^{\mathrm{A}}, d_{j}^{\mathrm{B}}-$ average delays for lane group $j$ at intersections A and B, separately [s/pcu];

$\alpha_{1}, \alpha_{2}$ - paired weighting factors;

$Q^{A}, Q^{B}$ - capacities of intersections $A$ and $B$, separately $[\mathrm{pcu} / \mathrm{h}]$; $\beta_{1}, \beta_{2}$ - paired weighting factors;

$\chi_{1}, \chi_{2}$ - paired weighting factors;

$\delta_{1}, \delta_{2}$ - paired weighting factors;

$Z$ - normal population, which represents the difference between normal populations $X$ and $Y$;

$X$ - normal population, which represents the performance measure of the reference scenario;

$Y$ - normal population, which represents the performance measure of the comparative scenario;

$t$ - statistic to testify whether null hypothesis $H_{0}: \mu=\mu_{0}$ is accepted or rejected;

$\mu$ - mean of normal population $Z$;

$\mu_{0}-$ known mean of normal population $Z$, and it equals 0 in this paper;

$\bar{z}$ - sample mean of normal population $Z$;

$s$ - sample standard deviation of normal population $Z$;

$n$ - sample size, which is the number of simulation runs in this paper;

$\sim$ - subject to;

$t(n-1)-t$-distribution with the freedom degree of $n-1$.

\section{Introduction}

Left-turn bays are often added to increase intersection capacity. Sometimes, left-turn bays are installed on the road segment between paired intersections. In the Highway Capacity Manual (2000), left-turn bays are generally viewed as exclusive left-turn lanes. Such a treatment does not consider the blockage and overflow of left-turn bays.

Many scholars have investigated the influences of leftturn or right-turn bays on traffic stream operations. Akçelik (1998) denoted that the saturation flow rate may drop for the approach with a left-turn bay at a signalized intersection. Tian and $\mathrm{Wu}$ (2006) considered the characteristics of traffic stream to formulate the probabilistic model to estimate the capacity of a signalized intersection approach with an added right-turn bay. Wu (2007) also used the theoretical-empirical method to calculate the capacity of a signalized intersection approach with an added left-turn bay. The estimation models were presented to calculate the lane utilization factors under the concern of intersection types (Lee et al. 2005). Zhang and Tong (2008) established the probabilistic model in which the left-turn bay length was an independent variable to estimate the capacities of the left-turn and through movements. When left-turn vehicles overflow on the left-turn bay, the estimation models were proposed to compute the discharge rate and capacity for the affected through movement (Osei-Asamoah et al. 2010). At a pretimed intersection, Yin et al. $(2010,2011)$ gave the theoretical models to estimate the delays for the left-turn and through movements with left-turn bays and protected left-turn operations. Some researchers investigated the potential safety and operational benefits of signalized intersections by adding left-turn bays (Tageldin et al. 2018) or adopted the extreme value theory to ana- 
lyse the conflicts at a signalized intersection with left-turn bays (Zheng et al. 2018). Most recently, Liu et al. (2019) proposed the probabilistic model to calculate the total capacity of a signalized intersection with left-turn bays by taking account into stochastic vehicle arrivals and signal timing plan.

On the determination of the required left-turn or rightturn bay length, some scholars developed the procedure for determining the left-turn bay length (Kikuchi et al. 2004). The appropriate turn bay lengths were examined for the case of which an approach lane was transformed into left-turn, through and right-turn lanes (Kikuchi et al. 2007). Kikuchi and Kronprasert (2010) proposed the analytical flowchart to calculate the left-turn bay lengths at signalized intersections in order to prevent the blockage and overflow of left-turn bays. Recently, Yang and Zhou (2011) proposed the coordination methodology to combine signal timing and the requirement of each component so that left-turn bays could be properly designed. Qi et al. $(2007,2012)$ utilized the simulation and analytical methods to determine the queue storage length and the deceleration length for left-turn bays.

The above-mentioned researches indicate that: the leftturn or right-turn bays have an important effect on traffic stream operations; and they should be well determined to enhance the intersection capacity and level of service. On the basis of the preliminary studies (Yao et al. 2011, 2012; Yao 2013a, 2013b, 2016), the joint optimization models are formulated for uncoordinated paired intersections with left-turn bays.

The remainder of the paper is arranged as below. The proposed methodology, which includes the basic assumptions, optimization models, model solutions and selection is developed in Section 1. A case study is demonstrated with the traffic data, sensitivity analysis and simulation tests in Section 2. To testify the reliabilities of the formulated models, the interval estimation and hypothesis testing are used to analyse the sample data from the simulated scenarios. Some discussions and suggestions are given with the procedure of model application in Section 3. The achievements and contributions are summarized at last.

\section{Proposed methodology}

\subsection{Basic assumptions}

According to the previous work (Yao et al. 2011; Yao 2013a, 2016), there is an optimal scheme of intersection layout and signal timing design, which can maximize capacity and minimize delay for an intersection with leftturn bays. Similarly, there should be an optimal scheme for each intersection as far as uncoordinated paired intersections with left-turn bays are concerned.

This paper focuses on uncoordinated paired intersections with left-turn bays. The Guidelines for Traffic Signals (RiLSA) (FGSV 2003) pointed out that Green Waves for motorized traffic are recommended for a traffic signal spacing of up to $750 \mathrm{~m}$, under particularly favourable conditions up to $1000 \mathrm{~m}$. There are two cases in which coordinated signals do not need to be considered. On the one hand, although the distance between paired intersections is shorter than a threshold, there is no movement controlled by coordinated signals when special management is adopted, such as one-way streets or three-leg intersections. On the other hand, paired intersections can be regarded as isolated intersections when the distance between them is longer than such a threshold. For these two cases, vehicles in each lane group randomly arrive at the intersection approach.

Figures 1 and 2 illustrate two cases of paired four-leg intersections with left-turn bays. In Case 1, the distance between paired intersections is shorter than a threshold; in Case 2, the distance is longer than such a threshold. It is assumed that right-turn traffic can go at any time under the condition of safety.

As shown in Figure 1a, the eastbound traffic at intersection $\mathrm{A}$, the westbound traffic at intersection $\mathrm{B}$, and the northbound and southbound left-turn traffic at intersections $\mathrm{A}$ and $\mathrm{B}$ are all prohibited. On the road segment between paired intersections, one exclusive right-turn lane, one through lane, one exclusive left-turn lane, and one left-turn bay are installed at each approach. One exclusive right-turn lane and two through lanes are installed at any northbound or southbound approach. In Figure 1b, there are two phases for the signal cycle of intersection A, that is, Phase 1 for the northbound and southbound traffic, and Phase 2 for the westbound traffic; there are two phases for the signal cycle of intersection B, that is, Phase 1 for the northbound and southbound traffic, and Phase 2 for the eastbound traffic. Case 1 can be extended to have more right-turn, through or left-turn lanes at the westbound approach of intersection $\mathrm{A}$ or at the eastbound approach of intersection $B$, or to have more right-turn or through lanes at the northbound or southbound approach of intersection A or B. A shared through-right lane can be installed at the southbound approach of intersection A or at the northbound approach of intersection B. A shared through-left lane can be installed at the westbound approach of intersection $A$ or at the eastbound approach of intersection B. Case 1 can also be simplified to the cases of prohibiting the southbound traffic at intersection $\mathrm{A}$ or the northbound traffic at intersection B, or three-leg intersections; and the signal phasing may be simpler.

As depicted in Figure 2a, a shared through-right lane, a through lane, an exclusive left-turn lane, and a left-turn bay are installed at each approach on the road segment between paired intersections, and a shared through-right lane, a through lane and an exclusive left-turn lane are installed at any other approach. In Figure 2b, there are four phases for the signal cycle of each intersection, i.e., Phase 1 for the westbound and eastbound through-right traffic, Phase 2 for the westbound and eastbound leftturn traffic, Phase 3 for the northbound and southbound through-right traffic, and Phase 4 for the northbound and southbound left-turn traffic. Case 2 can be extended to have two or more left-turn bays at each intersection. 


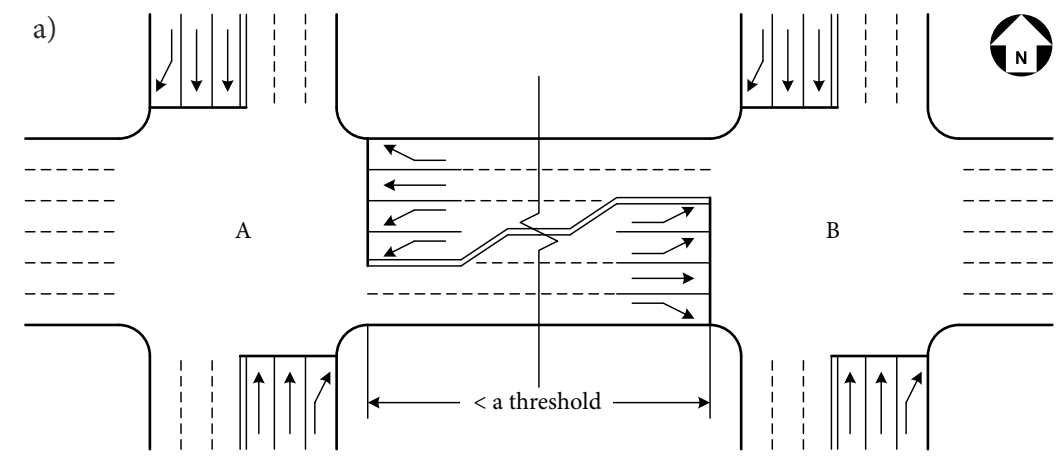

b)

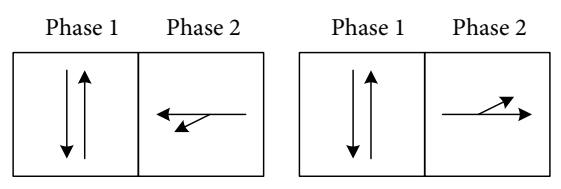

Intersection A

Intersection B

Figure 1. Case 1 of paired four-leg intersections: $a$ - geometry design; $b$ - signal phasing

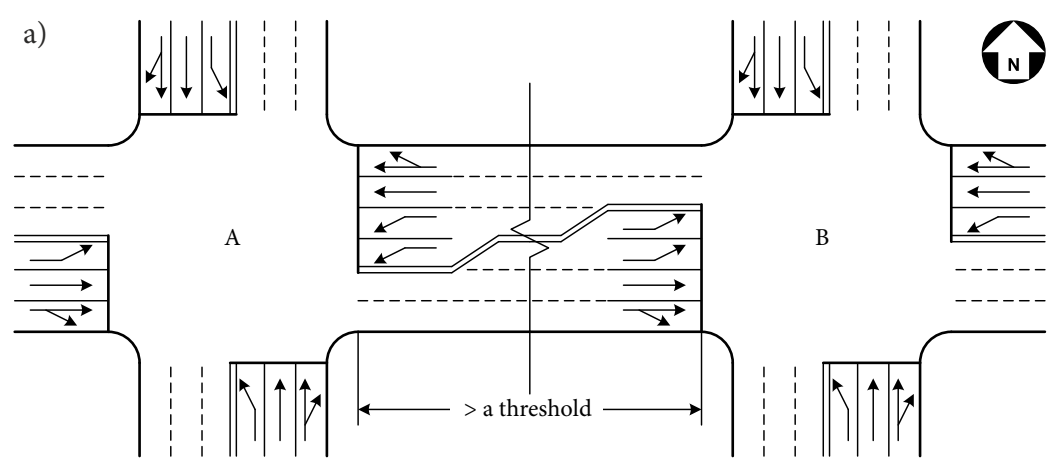

b)

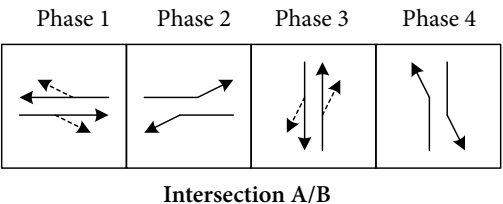

Figure 2. Case 2 of paired four-leg intersections: a - geometry design; $b$ - signal phasing

It can also be extended to have two or more through or exclusive left-turn lanes, or to have one or more exclusive right-turn lanes at each approach. In Case 2, an exclusive left-turn lane can be a shared through-left lane (at this time, the signal phasing may need to be adjusted), or there can be no shared through-left lane or exclusive left-turn lane at an approach. Case 2 can also be simplified to consider three-leg intersections or one-way streets, and the signal phasing may be simpler.

For Case 1, the through or right-turn movement randomly arrives at the southbound or northbound approach of intersection A or B since there is no upstream intersection; the right-turn, through or left-turn movement randomly arrives at the westbound approach of intersection A or the eastbound approach of intersection B since it originates from the right-turn movement at the upstream intersection, which is not controlled by any traffic signal. For Case 2, the right-turn, through or left-turn movement randomly arrives at the eastbound, southbound or northbound approach of intersection A or the westbound, southbound or northbound approach of intersection $B$ since there is no upstream intersection; the right-turn, through or left-turn movement randomly arrives at the westbound approach of intersection A or the eastbound approach of intersection $B$ since the distance between paired intersections is far enough. For any of these two cases, the left-turn bay lengths are not independent but interrelated for the westbound approach of intersection $A$ and the eastbound approach of intersection $B$, and the sum of the left-turn bay lengths should be less than or equal the road segment length for paired intersections.
According to actual situations, signal phasing can adopt the other scheme (e.g. split left-turn phasing) for each intersection in the above two cases. Considering uncoordinated signals, random arrivals can be assumed for each movement, the upstream filtering/metering adjustment factor and the uniform delay progression adjustment factor are both set to 1 . Assuming no initial queue in each lane group at the start of the analysis period, a value of 0 is used for the initial queue delay. Based on our previous study (Yao 2013a), the average phase lost time is set to $3.47 \mathrm{~s}$. Assuming that pretimed signals are used for paired intersections, a value of 0.5 is adopted for the incremental delay factor.

\subsection{Optimization models}

In general, maximizing the intersection capacity is pursued by transportation and traffic managers, while minimizing the travel delays are pursued by motorists or pedestrians.

Given the presence of left-turn bays (Akçelik 1998), the capacity of an intersection lane group can be estimated as:

$Q_{j}^{\eta}= \begin{cases}\frac{1}{C^{\eta}} \cdot\left(S_{f j}^{\eta} \cdot \sum_{i=1}^{n^{\eta}} \phi_{i j}^{\eta} \cdot g_{i}^{\eta}+\delta_{j}^{\eta} \cdot S_{s j}^{\eta} \cdot g_{j}^{\eta}\right), & \sum_{i=1}^{n^{\eta}} \phi_{i j}^{\eta} \cdot g_{i}^{\eta} \geq g_{j}^{\eta} ; \\ \frac{1}{C^{\eta}} \cdot\left(S_{f j}^{\eta}+\delta_{j}^{\eta} \cdot S_{s j}^{\eta}\right) \cdot \sum_{i=1}^{n^{\eta}} \phi_{i j}^{\eta} \cdot g_{i}^{\eta}, & \sum_{i=1}^{n^{\eta}} \phi_{i j}^{\eta} \cdot g_{i}^{\eta}<\widehat{g}_{j}^{\eta} .\end{cases}$

where: $\eta \in\{\mathrm{A}, \mathrm{B}\} ; C^{\eta}=\sum_{i=1}^{n^{\eta}} g_{i}^{\eta}+L^{\eta} ; \bar{g}_{j}^{\eta}=\frac{\bar{t}}{h} \cdot D_{j}^{\eta}$. 
The capacities of all the lane groups can be aggregated to obtain the capacity of the entire intersection. The latter is called intersection capacity and computed as:

$Q^{\eta}= \begin{cases}\frac{1}{C^{\eta}} \cdot \sum_{j=1}^{m^{\eta}}\left(S_{f j}^{\eta} \cdot \sum_{i=1}^{n^{\eta}} \phi_{i j}^{\eta} \cdot g_{i}^{\eta}+\delta_{j}^{\eta} \cdot S_{s j}^{\eta} \cdot \hat{g}_{j}^{\eta}\right), & \sum_{i=1}^{n^{\eta}} \phi_{i j}^{\eta} \cdot g_{i}^{\eta} \geq \hat{g}_{j}^{\eta} ; \\ \frac{1}{C^{\eta}} \cdot \sum_{j=1}^{m^{\eta}}\left(S_{f j}^{\eta}+\delta_{j}^{\eta} \cdot S_{s j}^{\eta}\right) \sum_{i=1}^{n^{\eta}} \phi_{i j}^{\eta} \cdot g_{i}^{\eta}, & \sum_{i=1}^{n^{\eta}} \phi_{i j}^{\eta} \cdot g_{i}^{\eta}<\widehat{g}_{j}^{\eta} .\end{cases}$

Based on the Highway Capacity Manual (2000) together with the above assumptions, the average vehicle delay for an intersection lane group can be given by:

$$
\begin{aligned}
& d_{j}^{\eta}=\frac{0.5 \cdot C^{\eta} \cdot\left(1-u_{j}^{\eta}\right)^{2}}{1-\min \left(1, x_{j}^{\eta}\right) \cdot u_{j}^{\eta}}+900 \cdot T \times \\
& \left(\left(x_{j}^{\eta}-1\right)+\sqrt{\left(x_{j}^{\eta}-1\right)^{2}+\frac{4 \cdot x_{j}^{\eta}}{Q_{j}^{\eta} \cdot T}}\right),
\end{aligned}
$$

where: $x_{j}^{\eta}=\frac{q_{j}^{\eta}}{Q_{j}^{\eta}} ; u_{j}^{\eta}=\frac{1}{C^{\eta}} \cdot \sum_{i=1}^{n^{\eta}} \phi_{i j}^{\eta} \cdot g_{i}^{\eta}$.

The average delay for the entire intersection is named intersection delay and can be obtained by the aggregation of the average delays for all the lane groups. The expression is:

$$
d^{\eta}=\frac{\sum_{j=1}^{m^{\eta}} q_{j}^{\eta} \cdot d_{j}^{\eta}}{\sum_{j=1}^{m^{\eta}} q_{j}^{\eta}}
$$

Signal timings often consider the safety of pedestrians crossing ( $\mathrm{Wu}, \mathrm{Li} 2015)$, the pedestrian effective green time for phase $i$ at intersection $\eta$ can be calculated as:

$$
\tilde{g}_{\min i}^{\eta}=\max _{j}\left(\frac{\varphi_{j}^{\eta} \cdot \phi_{i j}^{\eta} \cdot L_{p j}^{\eta}}{v_{p}}+7-I_{i}^{\eta}\right) .
$$

To satisfy traffic demands, the minimal cycle length for intersection $\eta$ is:

$$
C_{\min }^{\eta}=\max \left(\frac{L^{\eta}}{1-\min \left(Y^{\eta}, 0.9\right)}, 40\right) \text {, }
$$

where: $Y^{\eta}=\sum_{i=1}^{n^{\eta}} \max _{j}\left(\varphi_{j}^{\eta} \cdot \phi_{i j}^{\eta} \cdot y_{j}^{\eta}\right) ; y_{j}^{\eta}=\frac{q_{j}^{\eta}}{S_{f j}^{\eta}+S_{s j}^{\eta}}$.

In view of the Webster's formula (Akçelik 1998), the maximal cycle length for intersection $\eta$ can be estimated as:

$$
\mathrm{C}_{\max }^{\eta}=\min \left(\frac{1.5 \cdot L^{\eta}+5}{1-\min \left(Y^{\eta}, 0.9\right)}, 180\right) .
$$

Based on the principle of equal saturation degree, the minimal and maximal values of the effective green time for phase $i$ at intersection $\eta$ can be given by:

$$
\begin{aligned}
\hat{g}_{\min i}^{\eta} & =\frac{\min _{j}\left(\varphi_{j}^{\eta} \cdot \phi_{i j}^{\eta} \cdot y_{j}^{\eta}\right) \cdot\left(C_{\min }^{\eta}-L^{\eta}\right)}{Y^{\eta}} ; \\
g_{\max i}^{\eta} & =\frac{\max _{j}\left(\phi_{i j}^{\eta} \cdot y_{j}^{\eta}\right) \cdot\left(C_{\max }^{\eta}-L^{\eta}\right)}{Y^{\eta}} .
\end{aligned}
$$

When the crosswalk is relatively longer, the pedestrian effective green time may be greater. To guarantee the appropriate upper and lower limits, the minimal effective green time can be estimated as:

$$
g_{\min i}^{\eta}=\left\{\begin{array}{ll}
\tilde{g}_{\min i}^{\eta}, & \tilde{g}_{\min i}^{\eta}<g_{\max i}^{\eta} \\
\hat{g}_{\min i}^{\eta}, & \tilde{g}_{\min i}^{\eta} \geq g_{\max i}^{\eta}
\end{array} .\right.
$$

The phase effective green time should be within the appropriate upper and lower limits, i.e.:

$$
g_{\min i}^{\eta} \leq g_{i}^{\eta} \leq g_{\max i}^{\eta} .
$$

In order to avoid the blockage and overflow of a leftturn bay, the effective green time for the lane group should be greater than or equal to the queue full discharge time for the left-turn bay, that is:

$$
\sum_{i=1}^{n^{\eta}} \phi_{i j}^{\eta} \cdot g_{i}^{\eta} \geq \hat{g}_{j}^{\eta}, \delta_{j}^{\eta}=1 .
$$

In addition, the left-turn bay length should be nonnegative and less than a reasonable maximal value, i.e.:

$$
0 \leq D_{j}^{\eta} \leq D_{\max j}^{\eta}, \delta_{j}^{\eta}=1 .
$$

Finally, the sum of the left-turn bay lengths for the road segment between paired intersections should satisfy:

$$
\gamma_{k}^{\mathrm{A}} \cdot D_{k}^{\mathrm{A}}+\gamma_{l}^{\mathrm{B}} \cdot D_{l}^{\mathrm{B}} \leq D_{0},
$$

when $\gamma_{k}^{\mathrm{A}}=0$ and $\gamma_{l}^{\mathrm{B}}=0$, there is actually no such a constraint.

Four optimization models can be established to assign left-turn bay space and signal green time for paired intersections. When maximizing the intersection capacity, the optimization problem is to maximize Equation (2) under Equations (11)-(14), i.e.:

$$
\begin{aligned}
& \max Q^{\eta}=\frac{1}{C^{\eta}} \cdot \sum_{j=1}^{m^{\eta}}\left(S_{f j}^{\eta} \cdot \sum_{i=1}^{n^{\eta}} \phi_{i j}^{\eta} \cdot g_{i}^{\eta}+\frac{\bar{t}}{h} \cdot \delta_{j}^{\eta} \cdot S_{s j}^{\eta} \cdot D_{j}^{\eta}\right) \\
& \text { s.t. Equations }(11) \sim(14) .
\end{aligned}
$$

Similarly, when minimizing the intersection delay, the optimization problem is to minimize Equation (4) under Equations (11)-(14), i.e.:

$$
\min d^{\eta}=\frac{\sum_{j=1}^{m^{\eta}} q_{j}^{\eta} \cdot d_{j}^{\eta}}{\sum_{j=1}^{m^{\eta}} q_{j}^{\eta}}
$$

s.t. Equations $(11) \sim(14)$. 
If maximizing the intersection capacity and minimizing the intersection delay with the same constraints, the optimization problem becomes:

$$
\begin{aligned}
& \max Q^{\eta}=\frac{1}{C^{\eta}} \cdot \sum_{j=1}^{m^{\eta}}\left(S_{f j}^{\eta} \cdot \sum_{i=1}^{n^{\eta}} \phi_{i j}^{\eta} \cdot g_{i}^{\eta}+\frac{\bar{t}}{h} \cdot \delta_{j}^{\eta} \cdot S_{s j}^{\eta} \cdot D_{j}^{\eta}\right), \\
& \min d^{\eta}=\frac{\sum_{j=1}^{m^{\eta}} q_{j}^{\eta} \cdot d_{j}^{\eta}}{\sum_{j=1}^{m^{\eta}} q_{j}^{\eta}} \\
& \text { s.t. Equations }(11) \sim(14) .
\end{aligned}
$$

Additionally, when minimizing the total delay for paired intersections with the same constraints, the optimization model becomes:

$$
\begin{aligned}
& \min T D=d^{\mathrm{A}} \cdot q^{\mathrm{A}}+d^{\mathrm{B}} \cdot q^{\mathrm{B}} \\
& \text { s.t. Equations }(11)-(14),
\end{aligned}
$$

where: $d^{\mathrm{A}}=\frac{\sum_{j=1}^{m^{\mathrm{A}}} q_{j}^{\mathrm{A}} \cdot d_{j}^{\mathrm{A}}}{\sum_{j=1}^{m^{\mathrm{A}}} q_{j}^{\mathrm{A}}} ; d^{\mathrm{B}}=\frac{\sum_{j=1}^{m^{\mathrm{B}}} q_{j}^{\mathrm{B}} \cdot d_{j}^{\mathrm{B}}}{\sum_{j=1}^{m^{\mathrm{B}}} q_{j}^{\mathrm{B}}}$; $q^{\mathrm{A}}=\sum_{j=1}^{m^{\mathrm{A}}} q_{j}^{\mathrm{A}} ; q^{\mathrm{B}}=\sum_{j=1}^{m^{\mathrm{B}}} q_{j}^{\mathrm{B}}$.

\subsection{Model solutions and selection}

Equation (18) is a single-objective optimization problem. The fmincon function provided by MATLAB can be used to directly solve Equation (18). Equations (15)-(17) are all multi-objective optimization problems. They can be transformed into single-objective optimization problems. Then, they can be indirectly solved using the fmincon function. Two common methods which can convert several objectives into a single-objective are the weighted sum method and the multiplication/division method (Gan et al. 2005).

Equation (15) is a bi-objective optimization problem and can be transformed into a single-objective optimization problem to solve. Using the weighted sum method, Equation (15) can be converted into:

$$
\min \left(-\alpha_{1} \cdot Q^{\mathrm{A}}-\alpha_{2} \cdot Q^{\mathrm{B}}\right),
$$

when: $\alpha_{1}+\alpha_{2}=1 ; \alpha_{1}>0 ; \alpha_{2}>0$.

The bi-objective function in Equation (16) can also be transformed into a single-objective function, i.e.:

$$
\min \left(\beta_{1} \cdot d^{\mathrm{A}}+\beta_{2} \cdot d^{\mathrm{B}}\right),
$$

when: $\beta_{1}+\beta_{2}=1 ; \beta_{1}>0 ; \beta_{2}>0$.

Equation (17) indicates a four-objective optimization problem. As studied previously (Yao, 2013a), the capacityto-delay and delay-to-capacity ratios can measure the op- erations of an intersection. Thus, the transformed singleobjective function can be:

$$
\begin{gathered}
\min \left(-\chi_{1} \cdot \frac{Q^{\mathrm{A}}}{d^{\mathrm{A}}}-\chi_{2} \cdot \frac{Q^{\mathrm{B}}}{d^{\mathrm{B}}}\right), \\
\text { when: } \chi_{1}+\chi_{2}=1 ; \chi_{1}>0 ; \chi_{2}>0,
\end{gathered}
$$

or

$$
\min \left(\delta_{1} \cdot \frac{d^{\mathrm{A}}}{Q^{\mathrm{A}}}+\delta_{2} \cdot \frac{d^{\mathrm{B}}}{Q^{\mathrm{B}}}\right),
$$

when: $\delta_{1}+\delta_{2}=1 ; \delta_{1}>0 ; \delta_{2}>0$.

The transformed single-objective optimization models are solved by programming in MATLAB (https://www. mathworks.com). Capacity-to-delay ratio can evaluate the operations of an intersection (Yao 2013a). To evaluate the operations of paired intersections as a whole, the total capacity-to-delay ratio is defined and equals the sum of the capacity-to-delay ratios for intersections $\mathrm{A}$ and $\mathrm{B}$. The greater the capacity-to-delay ratio is, the better the corresponding scenario is for an intersection. Similarly, the greater the total capacity-to-delay ratio is, the better the corresponding scenario is for paired intersections as a whole. In this paper, we use the capacity-to-delay ratio and the total capacity-to-delay ratio to contrast different scenarios.

\section{Case study}

\subsection{Traffic data}

In Dalian City of China, we collected the field data during the morning peak-period at the uncoordinated paired intersections. One is Zhongshan Road and Youhao Street, the other is Changjiang Road and Youhao Street. The paired intersections are denoted by intersections $\mathrm{A}$ and B, separately.

Figure $3 \mathrm{a}$ shows the geometry design of the studied intersections. At intersection A, a left-turn bay with a length of $66 \mathrm{~m}$ is provided at the southbound approach. Accordingly, at intersection B, a left-turn bay with a length of $33 \mathrm{~m}$ is provided at the northbound approach. The road segment length for the paired intersections is $185 \mathrm{~m}$. Figure $3 \mathrm{~b}$ indicates the signal phasing for the studied intersections. The signal cycle of each intersection is divided into two phases. There is no signal to control the right-turn traffic. Consequently, all the movements randomly arrive at the intersection, that is to say, there is no movement, which needs to be controlled by coordinated signals. Therefore, coordinated signals do not need to be considered.

For the studied intersections, the saturation flow rates, the peak 5 min flow rates and the hourly volumes are listed in Table 1. Taking into account the fluctuation of traffic stream, the peak $5 \mathrm{~min}$ flow rates are adopted as the arrival flow rates to obtain the optimal intersection layout and signal timing scenario, while the hourly volumes are adopted to evaluate traffic stream operations under each scenario. 


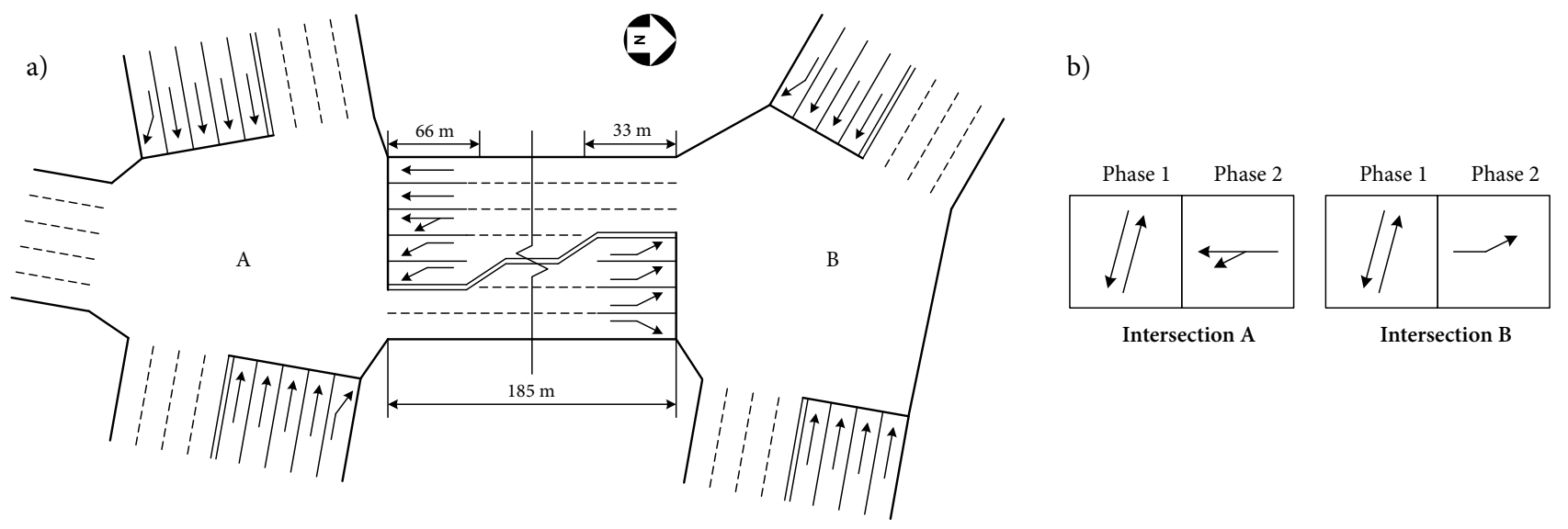

Figure 3. Geometry design and signal phasing for the study intersections: a - geometry design; b - signal phasing

Compared with the existing scenario denoted by $\mathrm{CP}$, a better scenario denoted by WP can be obtained using the Webster's formula (Quan 1989). Table 2 lists the left-turn bay lengths, signal timing parameters and performance measures for the existing and Webster's scenarios. Because the Webster's formula is only able to calculate the optimal cycle length and the green ratios, the left-turn bay lengths for $\mathrm{CP}$ are selected as those for $\mathrm{WP}$, and the lanes in the left-turn bays are regarded as the full- lanes for optimization. As shown in Table 2, the capacity-to-delay ratios for intersections $\mathrm{A}$ and $\mathrm{B}$ under WP are both greater than those CP. Thus, WP is better than CP.

\subsection{Sensitivity analysis}

Table 3 lists twenty-one typical objective functions. For these optimized scenarios, the left-turn bay lengths and the signal timing parameters are listed along with the performance measures in Table 4.

In view of Table 4, it is found that none of the optimized outcomes acquired by maximizing the weighted sum of intersection capacities (OP1 through OP5), by minimizing the weighted sum of intersection delays (OP6 through OP10) and by maximizing the weighted sum of capacityto-delay ratios (OP11 through OP15) are sensitive to the

Table 1 . Saturation flow rates, peak $5 \mathrm{~min}$ flow rates and hourly volumes

\begin{tabular}{|c|c|c|c|c|c|}
\hline Intersection & Traffic flow parameter $[\mathrm{pcu} / \mathrm{h}]$ & $\begin{array}{l}\text { Westbound } \\
\text { approach }\end{array}$ & $\begin{array}{c}\text { Eastbound } \\
\text { approach }\end{array}$ & $\begin{array}{l}\text { Northbound } \\
\text { approach }\end{array}$ & $\begin{array}{c}\text { Southbound } \\
\text { approach }\end{array}$ \\
\hline \multirow{4}{*}{ A } & full-lane saturation flow rate & 6743 & 7189 & - & 6556 \\
\hline & short-lane saturation flow rate & - & - & - & 1679 \\
\hline & peak 5 min flow rate & 3102 & 4278 & - & 2148 \\
\hline & hourly volume & 2563 & 3486 & - & 1751 \\
\hline \multirow{4}{*}{$\mathrm{B}$} & full-lane saturation flow rate & 5697 & 4713 & 3178 & - \\
\hline & short-lane saturation flow rate & - & - & 1567 & - \\
\hline & peak 5 min flow rate & 1812 & 2328 & 918 & - \\
\hline & hourly volume & 1228 & 1660 & 613 & - \\
\hline
\end{tabular}

Table 2. Involved parameters and performance indices for the existing (CP) and Webster's (WP) scenarios

\begin{tabular}{|c|c|c|c|c|c|c|c|c|c|c|}
\hline $\mathrm{CS}$ & Intersection & $D[\mathrm{~m}]$ & $g_{1}[\mathrm{~s}]$ & $g_{2}[\mathrm{~s}]$ & $C[\mathrm{~s}]$ & $Q[\mathrm{pcu} / \mathrm{h}]$ & $d[\mathrm{~s} / \mathrm{pcu}]$ & $x$ & $\frac{Q}{d}$ & $\sum \frac{Q}{d}$ \\
\hline \multirow{2}{*}{$\mathrm{CP}$} & A & 66.00 & 80.53 & 32.53 & 120.00 & 11435 & 19.94 & 0.84 & 573.38 & \multirow{2}{*}{1422.34} \\
\hline & $\mathrm{B}$ & 33.00 & 51.53 & 14.53 & 73.00 & 8217 & 9.68 & 0.71 & 848.96 & \\
\hline \multirow{2}{*}{ WP } & $\mathrm{A}$ & 66.00 & 69.57 & 30.49 & 107.00 & 11272 & 18.46 & 0.70 & 610.65 & \multirow{2}{*}{1467.31} \\
\hline & $\mathrm{B}$ & 33.00 & 38.13 & 14.93 & 60.00 & 7693 & 8.98 & 0.48 & 856.66 & \\
\hline
\end{tabular}

Notes: CS - scenario; $D$ - left-turn bay length; $g_{1}, g_{2}$ - effective green times for Phases 1 and 2, respectively; $C$ - cycle length; $Q$ - intersection capacity; $d$ - intersection delay; $x$ - intersection saturation degree; $\frac{Q}{d}$ - capacity-to-delay ratio; $\sum \frac{Q}{d}-$ total capacity-to-delay ratio; the amber time per phase is set to $3 \mathrm{~s}$; and the all-red time between successive phases is set to $2 \mathrm{~s}$. 
weighting factors of the objective functions. Nevertheless, comparing OP16 through OP20, the optimized outcomes (except the effective green time for Phase 2) acquired by minimizing the weighted sum of delay-to-capacity ratios are sensitive to the weighting factors of the objective functions. In view of the total capacity-to-delay ratios, OP19 is the optimal scenario among OP16 through OP20.

Table 3. Optimization scenarios and their objective functions

\begin{tabular}{|c|c|}
\hline $\begin{array}{l}\text { Optimization } \\
\text { scenario }\end{array}$ & Objective function \\
\hline OP1 & $\min \left(-0.1 \cdot Q^{\mathrm{A}}-0.9 \cdot Q^{\mathrm{B}}\right)$ \\
\hline OP2 & $\min \left(-0.3 \cdot Q^{\mathrm{A}}-0.7 \cdot Q^{\mathrm{B}}\right)$ \\
\hline OP3 & $\min \left(-0.5 \cdot Q^{\mathrm{A}}-0.5 \cdot Q^{\mathrm{B}}\right)$ \\
\hline $\mathrm{OP} 4$ & $\min \left(-0.7 \cdot Q^{\mathrm{A}}-0.3 \cdot Q^{\mathrm{B}}\right)$ \\
\hline OP5 & $\min \left(-0.9 \cdot Q^{\mathrm{A}}-0.1 \cdot Q^{\mathrm{B}}\right)$ \\
\hline OP6 & $\min \left(0.1 \cdot d^{\mathrm{A}}+0.9 \cdot d^{\mathrm{B}}\right)$ \\
\hline OP7 & $\min \left(0.3 \cdot d^{\mathrm{A}}+0.7 \cdot d^{\mathrm{B}}\right)$ \\
\hline OP8 & $\min \left(0.5 \cdot d^{\mathrm{A}}+0.5 \cdot d^{\mathrm{B}}\right)$ \\
\hline OP9 & $\min \left(0.7 \cdot d^{\mathrm{A}}+0.3 \cdot d^{\mathrm{B}}\right)$ \\
\hline OP10 & $\min \left(0.9 \cdot d^{\mathrm{A}}+0.1 \cdot d^{\mathrm{B}}\right)$ \\
\hline OP0 & $\min \left(d^{\mathrm{A}} \cdot q^{\mathrm{A}}+d^{\mathrm{B}} \cdot q^{\mathrm{B}}\right)$ \\
\hline OP11 & $\min \left(-0.1 \cdot \frac{Q^{\mathrm{A}}}{d^{\mathrm{A}}}-0.9 \cdot \frac{Q^{\mathrm{B}}}{d^{\mathrm{B}}}\right)$ \\
\hline OP12 & $\min \left(-0.3 \cdot \frac{Q^{\mathrm{A}}}{d^{\mathrm{A}}}-0.7 \cdot \frac{Q^{\mathrm{B}}}{d^{\mathrm{B}}}\right)$ \\
\hline OP13 & $\min \left(-0.5 \cdot \frac{Q^{\mathrm{A}}}{d^{\mathrm{A}}}-0.5 \cdot \frac{Q^{\mathrm{B}}}{d^{\mathrm{B}}}\right)$ \\
\hline OP14 & $\min \left(-0.7 \cdot \frac{Q^{\mathrm{A}}}{d^{\mathrm{A}}}-0.3 \cdot \frac{Q^{\mathrm{B}}}{d^{\mathrm{B}}}\right)$ \\
\hline OP15 & $\min \left(-0.9 \cdot \frac{Q^{\mathrm{A}}}{d^{\mathrm{A}}}-0.1 \cdot \frac{Q^{\mathrm{B}}}{d^{\mathrm{B}}}\right)$ \\
\hline OP16 & $\min \left(0.1 \cdot \frac{d^{\mathrm{A}}}{Q^{\mathrm{A}}}+0.9 \cdot \frac{d^{\mathrm{B}}}{\mathrm{Q}^{\mathrm{B}}}\right)$ \\
\hline OP17 & $\min \left(0.3 \cdot \frac{d^{\mathrm{A}}}{Q^{\mathrm{A}}}+0.7 \cdot \frac{d^{\mathrm{B}}}{\mathrm{Q}^{\mathrm{B}}}\right)$ \\
\hline OP18 & $\min \left(0.5 \cdot \frac{d^{\mathrm{A}}}{Q^{\mathrm{A}}}+0.5 \cdot \frac{d^{\mathrm{B}}}{Q^{\mathrm{B}}}\right)$ \\
\hline OP19 & $\min \left(0.7 \cdot \frac{d^{\mathrm{A}}}{Q^{\mathrm{A}}}+0.3 \cdot \frac{d^{\mathrm{B}}}{Q^{\mathrm{B}}}\right)$ \\
\hline OP20 & $\min \left(0.9 \cdot \frac{d^{\mathrm{A}}}{\mathrm{Q}^{\mathrm{A}}}+0.1 \cdot \frac{d^{\mathrm{B}}}{\mathrm{Q}^{\mathrm{B}}}\right)$ \\
\hline
\end{tabular}

As listed in Table 1, the peak 5 min flow rates are given together with the hourly volumes, and these values are regarded as the baselines of traffic demands. Figure 4 illustrates the variation curve of the optimal left-turn bay lengths acquired by minimizing the weighted sum of the capacity-to-delay ratios versus the growth rate of traffic demand. Here, the growth rates of the peak 5 min flow rates are identical to those of the hourly volumes for all the movements; and the two weighting factors are equal to 1 . It can be seen that the optimized length of each leftturn bay may increase as the traffic demand increases. When the traffic demands increase by $15 \%$, the sum of the left-turn bay lengths equals the road segment length. When the traffic demands increase by $16 \%$, the optimized results will vary, but the sum of the left-turn bay lengths still equals the road segment length.

\subsection{Simulation tests}

To investigate the influences of different scenarios on traffic stream operations, we utilize the traffic simulation tool VISSIM to test the seven scenarios listed in Table 5. For each scenario, the left-turn bay lengths, displayed green times and cycle lengths are given. OP0 is actually identical to OP9 because of the same value of each parameter at a given level of precision.

In VISSIM (https://www.ptvgroup.com/en/solutions/ products(ptv-vissim), the vehicle inputs adopt the hourly volumes for all the lane groups. In simulation, the simulation period of $4500 \mathrm{~s}$, the warm-up time of $900 \mathrm{~s}$, and the mode of multi-run are adopted. Because it is recommended by the PTV VISSIM User Manuals (PTV 2012, 2014) that the number of simulation runs should adopt $5 . . .20$ for static assignment, the default value of 10 is used for the number of simulation runs to balance the calculation precision and workload in this paper. Specifically, the random seeds range from 5 to 95 with an increment of 10 .

In this study, the Wiedemann 74 model is selected to describe car following behaviour (PTV 2012, 2014).

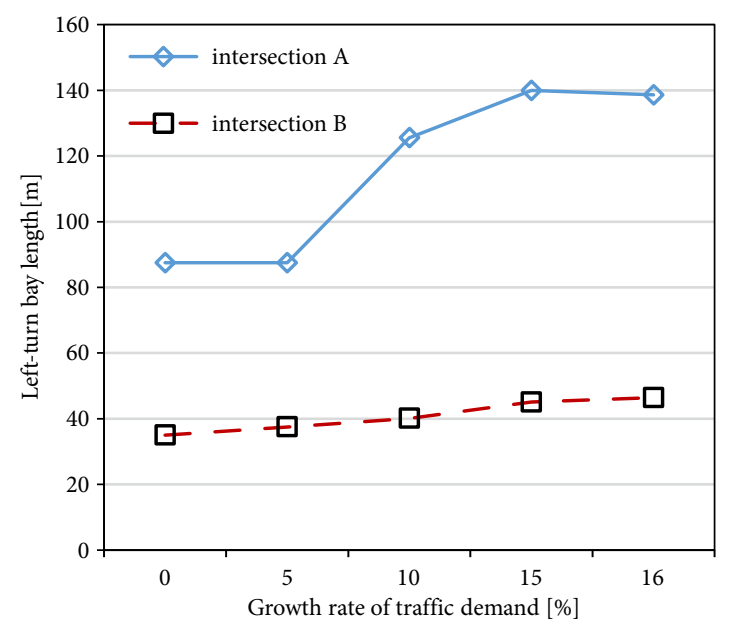

Figure 4. Variation curve of left-turn bay length versus growth rate of traffic demand 
Table 4. Optimization results under different scenarios

\begin{tabular}{|c|c|c|c|c|c|c|c|c|c|c|}
\hline $\begin{array}{l}\text { Optimization } \\
\text { scenario }\end{array}$ & Intersection & $D[\mathrm{~m}]$ & $g_{1}[\mathrm{~s}]$ & $g_{2}[\mathrm{~s}]$ & $C[\mathrm{~s}]$ & $Q[\mathrm{pcu} / \mathrm{h}]$ & $d[\mathrm{~s} / \mathrm{pcu}]$ & $x$ & $\frac{Q}{d}$ & $\sum \frac{Q}{d}$ \\
\hline \multirow{2}{*}{ OP1 } & $\mathrm{A}$ & 87.50 & 69.53 & 29.17 & 105.64 & 11444 & 17.76 & 0.77 & 644.21 & \multirow{2}{*}{1612.96} \\
\hline & $\mathrm{B}$ & 35.00 & 30.44 & 11.67 & 49.04 & 7589 & 7.83 & 0.57 & 968.75 & \\
\hline \multirow{2}{*}{ OP2 } & $\mathrm{A}$ & 87.50 & 69.53 & 29.17 & 105.64 & 11444 & 17.76 & 0.77 & 644.21 & \multirow{2}{*}{1612.96} \\
\hline & B & 35.00 & 30.44 & 11.67 & 49.04 & 7589 & 7.83 & 0.57 & 968.75 & \\
\hline \multirow{2}{*}{ OP3 } & $\mathrm{A}$ & 87.50 & 69.53 & 29.17 & 105.64 & 11444 & 17.76 & 0.77 & 644.21 & \multirow{2}{*}{$1,612.96$} \\
\hline & B & 35.00 & 30.44 & 11.67 & 49.04 & 7589 & 7.83 & 0.57 & 968.75 & \\
\hline \multirow{2}{*}{ OP4 } & A & 87.50 & 69.53 & 29.17 & 105.64 & 11444 & 17.76 & 0.77 & 644.21 & \multirow{2}{*}{1612.96} \\
\hline & B & 35.00 & 30.44 & 11.67 & 49.04 & 7589 & 7.83 & 0.57 & 968.75 & \\
\hline \multirow{2}{*}{ OP5 } & $\mathrm{A}$ & 87.50 & 69.53 & 29.17 & 105.64 & 11444 & 17.76 & 0.77 & 644.21 & \multirow{2}{*}{1612.96} \\
\hline & $\mathrm{B}$ & 35.00 & 30.44 & 11.67 & 49.04 & 7589 & 7.83 & 0.57 & 968.75 & \\
\hline \multirow{2}{*}{ OP6 } & $\mathrm{A}$ & 87.50 & 64.47 & 29.17 & 100.57 & 11318 & 17.49 & 0.76 & 647.13 & \multirow{2}{*}{1605.09} \\
\hline & B & 35.00 & 28.84 & 11.67 & 47.44 & 7494 & 7.82 & 0.58 & 957.96 & \\
\hline \multirow{2}{*}{ OP7 } & $\mathrm{A}$ & 87.50 & 64.46 & 29.17 & 100.57 & 11318 & 17.49 & 0.76 & 647.14 & \multirow{2}{*}{1605.08} \\
\hline & $\mathrm{B}$ & 35.00 & 28.83 & 11.67 & 47.44 & 7494 & 7.82 & 0.58 & 957.94 & \\
\hline \multirow{2}{*}{ OP8 } & A & 87.50 & 64.46 & 29.17 & 100.57 & 11318 & 17.49 & 0.76 & 647.14 & \multirow{2}{*}{1605.08} \\
\hline & B & 35.00 & 28.83 & 11.67 & 47.44 & 7494 & 7.82 & 0.58 & 957.94 & \\
\hline \multirow{2}{*}{ OP9 } & A & 87.50 & 64.47 & 29.17 & 100.57 & 11318 & 17.49 & 0.76 & 647.13 & \multirow{2}{*}{1605.09} \\
\hline & B & 35.00 & 28.84 & 11.67 & 47.44 & 7494 & 7.82 & 0.58 & 957.96 & \\
\hline \multirow{2}{*}{ OP10 } & A & 87.50 & 64.46 & 29.17 & 100.57 & 11318 & 17.49 & 0.76 & 647.14 & \multirow{2}{*}{1605.13} \\
\hline & B & 35.00 & 28.84 & 11.67 & 47.45 & 7494 & 7.82 & 0.58 & 957.98 & \\
\hline \multirow{2}{*}{ OP11 } & A & 87.50 & 65.41 & 29.17 & 101.51 & 11343 & 17.53 & 0.75 & 646.99 & \multirow{2}{*}{1615.74} \\
\hline & B & 35.00 & 30.44 & 11.67 & 49.04 & 7589 & 7.83 & 0.57 & 968.75 & \\
\hline & A & 87.50 & 65.41 & 29.17 & 101.51 & 11343 & 17.53 & 0.75 & 646.99 & $1610^{-1}$ \\
\hline U12 & B & 35.00 & 30.44 & 11.67 & 49.04 & 7589 & 7.83 & 0.57 & 968.75 & $1015 . / 4$ \\
\hline $\mathrm{QD} 12$ & A & 87.50 & 65.41 & 29.17 & 101.51 & 11343 & 17.53 & 0.75 & 646.99 & 161574 \\
\hline $0 \% 13$ & B & 35.00 & 30.44 & 11.67 & 49.04 & 7589 & 7.83 & 0.57 & 968.75 & $1015 . / 4$ \\
\hline OD14 & A & 87.50 & 65.41 & 29.17 & 101.51 & 11343 & 17.53 & 0.75 & 646.99 & 161571 \\
\hline 0114 & B & 35.00 & 30.44 & 11.67 & 49.04 & 7589 & 7.83 & 0.57 & 968.75 & 1015.14 \\
\hline & A & 87.50 & 65.41 & 29.17 & 101.51 & 11343 & 17.53 & 0.75 & 646.99 & 10157 \\
\hline U11 & B & 35.00 & 30.44 & 11.67 & 49.04 & 7589 & 7.83 & 0.57 & 968.75 & 1015.14 \\
\hline OD1 & A & 18.02 & 20.98 & 29.17 & 57.09 & 8647 & 300.08 & 1.32 & 28.81 & 2020 \\
\hline 010 & B & 18.22 & 27.94 & 11.67 & 46.55 & 7250 & 8.11 & 0.61 & 893.42 & 922.23 \\
\hline 7 & A & 18.37 & 55.99 & 29.17 & 92.10 & 10658 & 18.05 & 0.80 & 590.33 & $140^{-0}$ \\
\hline UP1 & B & 20.21 & 26.26 & 11.87 & 45.06 & 7137 & 8.13 & 0.60 & 878.19 & 1408.32 \\
\hline OD10 & A & 18.15 & 32.95 & 29.17 & 69.05 & 9564 & 39.35 & 1.02 & 243.07 & 1140 \\
\hline 010 & B & 18.26 & 30.44 & 11.67 & 49.05 & 7411 & 8.19 & 0.64 & 905.37 & 1140.45 \\
\hline OP10 & A & 18.35 & 50.59 & 29.17 & 86.70 & 10454 & 17.99 & 0.83 & 580.96 & 148637 \\
\hline (1) & B & 18.26 & 30.44 & 11.67 & 49.05 & 7411 & 8.19 & 0.64 & 905.41 & 1400.07 \\
\hline & A & 18.42 & 56.76 & 29.17 & 92.87 & 10685 & 18.09 & 0.81 & 590.78 & 120005 \\
\hline$O P 20$ & B & 18.08 & 17.00 & 11.67 & 35.61 & 6277 & 8.86 & 0.74 & 708.27 & 1299.00 \\
\hline & A & 87.50 & 64.47 & 29.17 & 100.57 & 11318 & 17.49 & 0.76 & 647.13 & 160500 \\
\hline UPU & B & 35.00 & 28.83 & 11.67 & 47.44 & 7494 & 7.82 & 0.58 & 957.94 & 1003.08 \\
\hline
\end{tabular}

Notes:

- the lengths of the crosswalks for Phases 1 and 2 are 21.2 and $32.6 \mathrm{~m}$, respectively at intersection A; they are 10.7 and $11.6 \mathrm{~m}$, separately at intersection $B$;

- the intergreen time between successive phases is set to $5 \mathrm{~s}$. 
Table 5. Left-turn bay length and signal timing parameters for seven simulation scenarios

\begin{tabular}{|c|c|c|c|c|c|}
\hline \multirow{2}{*}{ Scenario } & \multirow{2}{*}{ Intersection } & \multirow{2}{*}{ Left-turn bay length $[\mathrm{m}]$} & \multicolumn{2}{|c|}{ Displayed green time $[\mathrm{s}]$} & \multirow{2}{*}{ Cycle length $[\mathrm{s}$} \\
\hline & & & Phase 1 & Phase 2 & \\
\hline \multirow{2}{*}{$\mathrm{CP}$} & A & 66.0 & 79 & 31 & 120 \\
\hline & $\mathrm{B}$ & 33.0 & 50 & 13 & 73 \\
\hline \multirow{2}{*}{ WP } & A & 66.0 & 68 & 29 & 107 \\
\hline & $\mathrm{B}$ & 33.0 & 37 & 13 & 60 \\
\hline \multirow{2}{*}{ OP4 } & A & 87.5 & 68 & 28 & 106 \\
\hline & $\mathrm{B}$ & 35.0 & 29 & 10 & 49 \\
\hline \multirow{2}{*}{ OP9 } & $\mathrm{A}$ & 87.5 & 63 & 28 & 101 \\
\hline & $\mathrm{B}$ & 35.0 & 27 & 10 & 47 \\
\hline \multirow{2}{*}{ OP14 } & $\mathrm{A}$ & 87.5 & 64 & 28 & 102 \\
\hline & $\mathrm{B}$ & 35.0 & 29 & 10 & 49 \\
\hline \multirow{2}{*}{ OP19 } & $\mathrm{A}$ & 18.4 & 49 & 28 & 87 \\
\hline & $\mathrm{B}$ & 18.3 & 29 & 10 & 49 \\
\hline \multirow{2}{*}{ OP0 } & $\mathrm{A}$ & 87.5 & 63 & 28 & 101 \\
\hline & $\mathrm{B}$ & 35.0 & 27 & 10 & 47 \\
\hline
\end{tabular}

Note: the displayed green time means the duration of the green indication for a phase, and equals the sum of the effective green time and the start-up lost time minus the amber time for the phase.

Table 6. Settings of driving behaviour parameters and lane widths

\begin{tabular}{|c|c|c|c|c|}
\hline \multicolumn{2}{|c|}{ Link / Connector type } & $\begin{array}{c}\text { Average standstill } \\
\text { distance }[\mathrm{m}]\end{array}$ & $\begin{array}{c}\text { Additive part of safety } \\
\text { distance }[\mathrm{m}]\end{array}$ & $\begin{array}{l}\text { Multiplicative part of safety } \\
\text { distance }[\mathrm{m}]\end{array}$ \\
\hline \multirow{4}{*}{ Intersection A } & eastbound through & 2.00 & 2.45 & 3.45 \\
\hline & southbound left-turn & 2.00 & 2.61 & 3.61 \\
\hline & southbound through & 2.00 & 2.66 & 3.66 \\
\hline & westbound through & 2.00 & 2.59 & 3.59 \\
\hline \multirow{3}{*}{ Intersection B } & eastbound through & 2.00 & 2.74 & 3.74 \\
\hline & westbound through & 2.00 & 2.92 & 3.92 \\
\hline & northbound left-turn & 2.00 & 2.72 & 3.72 \\
\hline \multicolumn{2}{|c|}{ Any right-turn } & 2.00 & 2.55 & 3.55 \\
\hline \multicolumn{2}{|c|}{ Others } & 2.00 & 2.00 & 3.00 \\
\hline \multicolumn{3}{|c|}{ Lane attribute } & \multicolumn{2}{|c|}{ Lane width from inner to outer [m] } \\
\hline \multirow{3}{*}{\multicolumn{2}{|c|}{ Intersection $\mathrm{A}$}} & eastbound approach & \multicolumn{2}{|c|}{$3.00 / 3.30 / 3.50 / 3.40 / 3.50$} \\
\hline & & southbound approach & \multicolumn{2}{|c|}{$2.60 / 2.80 / 3.10 / 3.20 / 3.30$} \\
\hline & & westbound approach & \multicolumn{2}{|c|}{$2.90 / 3.00 / 3.30 / 3.30 / 4.00$} \\
\hline \multirow{3}{*}{\multicolumn{2}{|c|}{ Intersection B }} & eastbound approach & \multicolumn{2}{|c|}{$2.70 / 3.00 / 3.00 / 3.50$} \\
\hline & & westbound approach & \multicolumn{2}{|c|}{$2.70 / 3.10 / 3.30 / 3.50$} \\
\hline & & northbound approach & \multicolumn{2}{|c|}{$3.00 / 3.30 / 3.20 / 3.50$} \\
\hline \multirow{2}{*}{\multicolumn{2}{|c|}{ Road segment between intersections A and B }} & from $\mathrm{A}$ to $\mathrm{B}$ & \multicolumn{2}{|r|}{3.30} \\
\hline & & from $\mathrm{B}$ to $\mathrm{A}$ & \multicolumn{2}{|r|}{3.40} \\
\hline \multicolumn{3}{|c|}{ Others } & \multicolumn{2}{|r|}{3.50} \\
\hline
\end{tabular}

To simulate the measured saturation flow rates, the settings of driving behaviour parameters are listed in Table 6 together with the settings of lane widths. Because the approach lane widths are all different at each intersection, the saturation flow rates for these approach lanes are all various by observation. Therefore, not only the additive parts of safety distance but also the multiplicative parts of safety distance are all different for the approach lanes at each intersection.
In each simulated scenario, the distance between the starting and ending sections is $150 \mathrm{~m}$ for each travel time section. Each queue counter locates at the stop-line. The travel time section can provide the delay for the corresponding movement. The queue counter can provide the queue length for the corresponding approach.

In Figure 5, the average and standard deviation, and the upper and lower limits of the confidence interval are illustrated for the average vehicle delay, number of stops 
and speed in the network, and the average vehicle delay at the intersections under all the simulated scenarios. The former three indices are obtained from the network performance evaluation, and the latter one is gotten from the travel time sections. Here, the confidence level is $95 \%$, and the sample size is 10 , which is the number of simulation runs. As shown, the standard deviation of the average delay, number of stops or speed is far less than its average, or the upper or lower limit of the confidence interval, and its average falls into its confidence interval. Using the interval estimation for the average delay, number of stops or speed (Sun 2014), the expected confidence interval is assumed to be 1.50 times as much as the standard deviation, and the calculated confidence interval is 1.43 times as much as the standard deviation based on the sample data. Because the calculated value of the confidence interval is less than its expected value (Sun 2014), it can be proven that 10 is effective and sufficient for the number of simulation runs.

Any two of the seven simulated scenarios are paired, the total of twenty-one pairs can be gotten. Using the Student's $t$-test (Zhang, Gao 1997), the left and right critical

a)

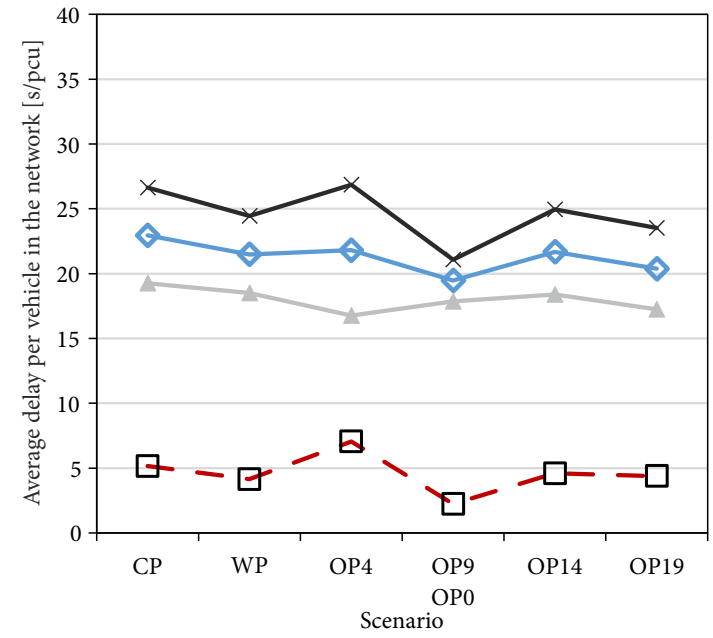

c)

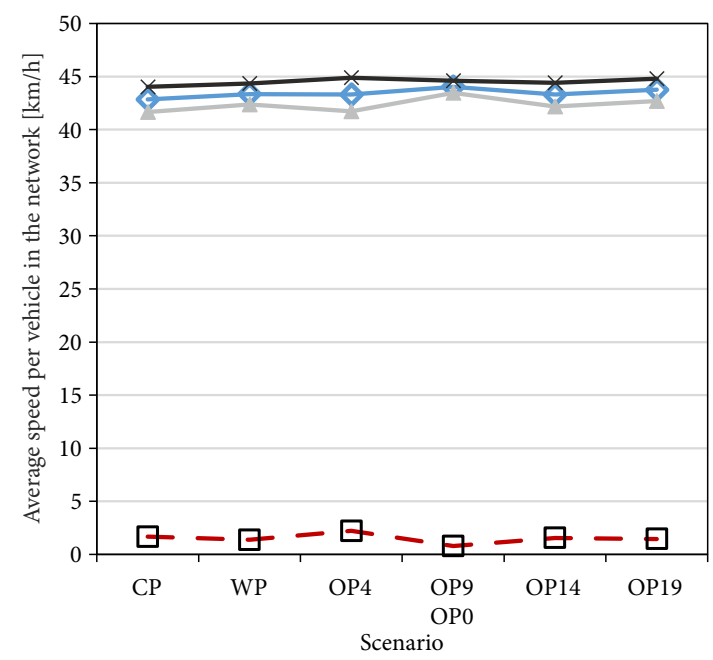

$t$-values can be obtained from the $t$-distribution table, and the $t$-value of the statistic about the mean of the difference between paired scenarios for a given index can be computed as:

$$
t=\frac{\bar{z}-\mu_{0}}{\frac{s}{\sqrt{n}}} \sim t(n-1),
$$

where: $Z=X-Y$.

At the significance level $\alpha$, if $t \leq-t_{\alpha}(n-1)$, the alternative hypothesis $H_{1}: \mu<\mu_{0}$ should be accepted; if $t \geq t_{\alpha}(n-1)$, the alternative hypothesis $\bar{H}_{1}: \mu>\mu_{0}$ should be accepted; otherwise, the null hypothesis $H_{0}: \mu=\mu_{0}$ should be accepted. Here, $-t_{\alpha}(n-1)$ and $t_{\alpha}(n-1)$ are the left and right critical $t$-values at the significance level $\alpha$ and with the freedom degree of $n-1$. In this case, the significance and confidence levels are $\alpha$ and $1-\alpha$, respectively.

The less the average delay is, the less the average number of stops is, or the higher the average speed is, the better the corresponding scenario is for traffic stream operations.

b)

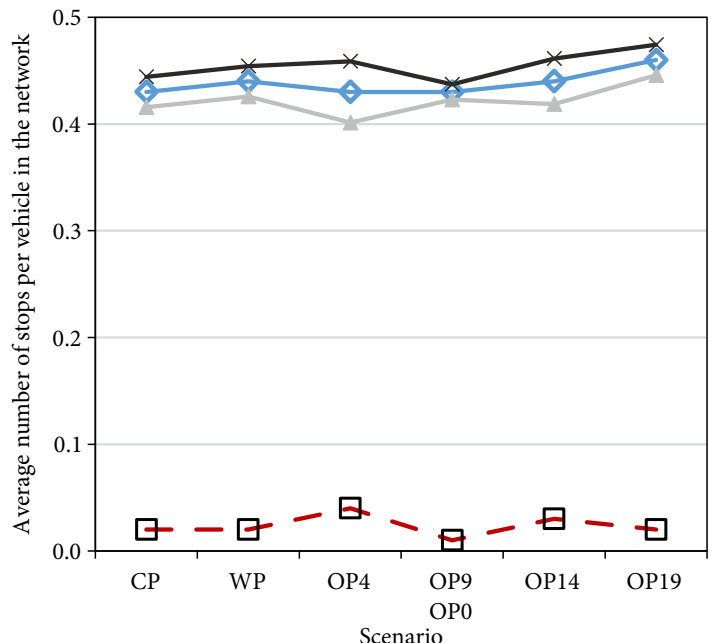

d)

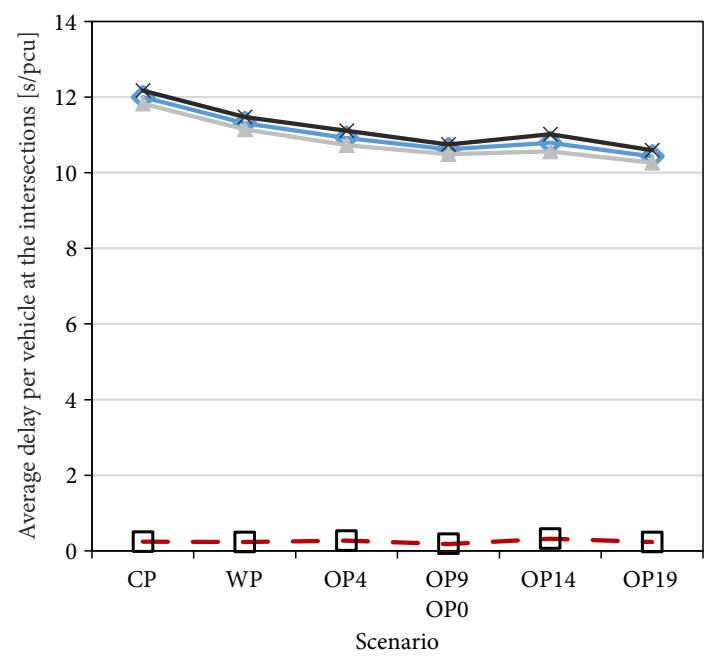

$\smile$ average $\square-$ standard deviation $\quad-\quad$ lower limit of confidence interval $\quad \longleftarrow$ upper limit of confidence interval

Figure 5. Comparison of the statistical indices for different scenarios: $a$ - average delay per vehicle in the network; $b$ - average number of stops per vehicle in the network; $\mathrm{c}$ - average speed per vehicle in the network; $\mathrm{d}$ - average delay per vehicle at the intersections 


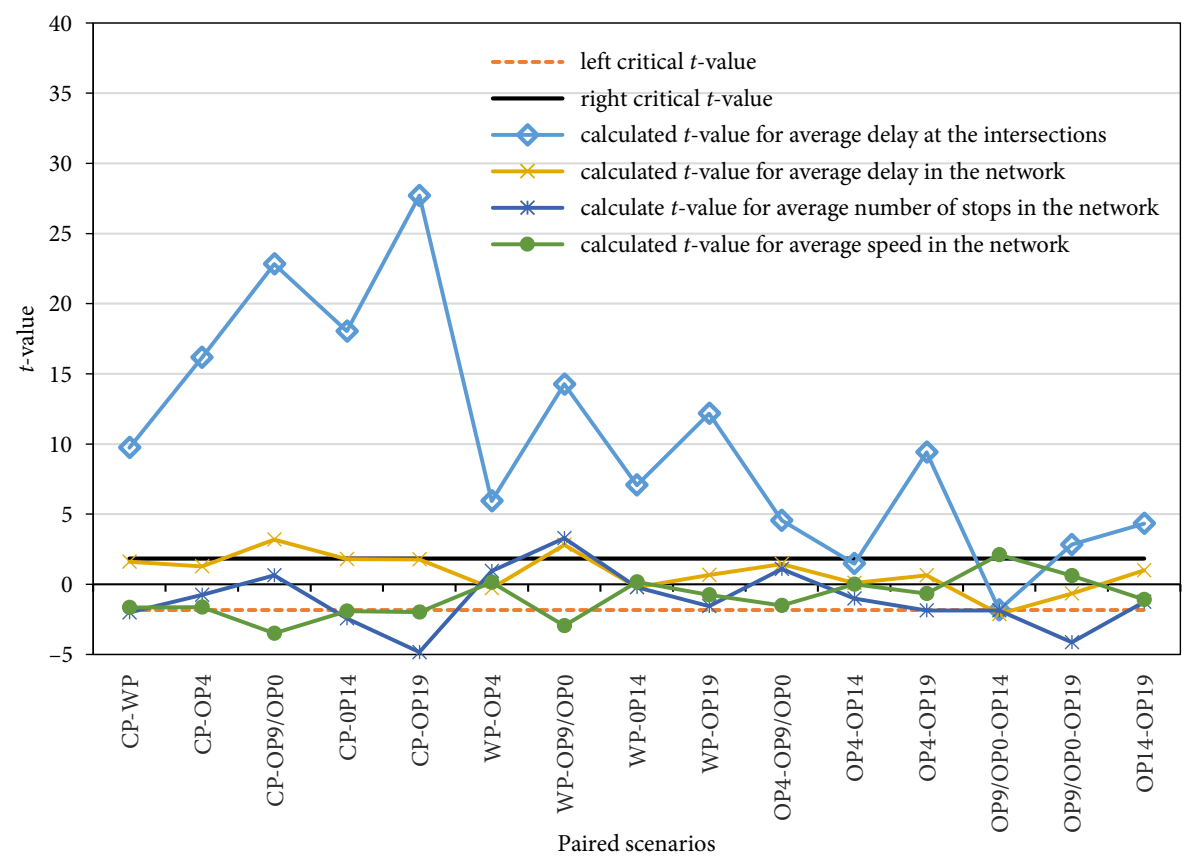

Figure 6. Results of Student's $t$-tests for different indices

Figure 6 illustrates the outcomes of Student's $t$-tests at the significance level $5 \%$ for the average delay at the intersections, and the average delay, number of stops and speed in the network. The results reveal that:

1) according to the average delay at the intersections, WP, OP4, OP9/OP0, OP14 and OP19 are all better than CP, and OP4, OP9/OP0, OP14 and OP19 are all better than WP at the significance level 5\%; OP9/OP0 and OP19 are both better than OP4, and OP19 is better than OP9/OP0 and OP14 at the significance level 5\%;

2) based on the average delay in the network, OP9/ OP0 is better than CP and WP at the significance level 5\%;

3) in view of the average number of stops in the network, OP9/OP0 is better than WP at the significance level 5\%;

4) on the basis of the average speed in the network, OP9/OP0, OP14 and OP19 is better than CP, and OP9/OP0 is better than WP and OP14 at the significance level $5 \%$.

Figure 7 illustrates the vehicle throughputs and average delays under the aforementioned seven simulated scenarios. These values are the means from 10 simulation runs. The results indicate that:

1) the vehicle throughput for each movement or intersection is not sensitive to signal timing scenario;

2) the average delay for the eastbound or westbound approach is not sensitive to signal timing scenario, whereas the average delay for the southbound or northbound approach is very sensitive to signal timing scenario;

3) the variation of the average delay for the entire intersection depends on that for the southbound or northbound approach;
4) on the whole, $\mathrm{CP}$ is the worst, $\mathrm{WP}$ is the second place, OP4, OP9/OP0, OP14, and OP19 are all better than CP and WP.

It is proven that the reasonable assignment of lane space on the road segment between paired intersections is important for traffic stream operations.

As shown in Table 7, the intersection performance measures are presented for these scenarios. According to the vehicle throughput-to-average delay ratios, the optimal sequences of these scenarios are OP19, OP9/OP0, OP14, OP4, WP, and CP for intersection A, and OP9/OP0, OP19, OP4, OP14, WP, and CP for intersection B from the best to the worst. For the paired intersections as a whole, the sequence is OP19, OP9/OP0, OP4, OP14, WP, and CP.

Figure 8 depicts the total increment of the average queue lengths in $5 \mathrm{~min}$ for each intersection under the simulated scenarios. These values are the means from 10 simulation runs. Considering $\mathrm{CP}$ as a baseline, the other scenarios are compared. For example, "WP-CP, A" is the sum of the average queue lengths for all the approaches

Table 7. Intersection performance measures for seven simulated scenarios

\begin{tabular}{|l|c|c|c|}
\hline \multirow{2}{*}{ Scenario } & \multicolumn{3}{|c|}{ Vehicle throughput-to-average delay ratio } \\
\cline { 2 - 4 } & Intersection A & Intersection B & Total \\
\hline CP & 565.01 & 714.83 & 1279.84 \\
\hline WP & 598.98 & 744.41 & 1343.39 \\
\hline OP4 & 614.49 & 813.86 & 1428.35 \\
\hline OP9/OP0 & 634.43 & 828.05 & 1462.48 \\
\hline OP14 & 624.74 & 799.84 & 1424.58 \\
\hline OP19 & 647.63 & 827.39 & 1475.02 \\
\hline
\end{tabular}


under WP minus that under $\mathrm{CP}$ during a given time interval for intersection A. As shown in Figure 8, compared with $\mathrm{CP}$, the other scenarios make the 5 min average queue lengths shorten in the most cases. Based on the mean of the average queue lengths in $5 \mathrm{~min}$, the five optimized scenarios are all better than the CP and WP. On the whole, the sequence of these scenarios is OP9/OP0, OP14, OP19, OP4, WP, and CP from the best to the worst.

Table 8 lists the average, maximum, minimum and standard deviation of the average delay, stops, speed, stopped delay, active vehicles and arrived vehicles under the above-mentioned seven simulated scenarios. These values originate from the network performance evaluation. It can be seen that:

1) the standard deviation of any performance measure is far less than its average, maximum or minimum for each scenario;

2) according to the average values, the average delay will decrease as the average speed increases;

3) based on the average delay and speed, the sequence from the best to the worst is OP9/OP0, OP19, WP, OP14, OP4 and CP for the seven scenarios.

a)

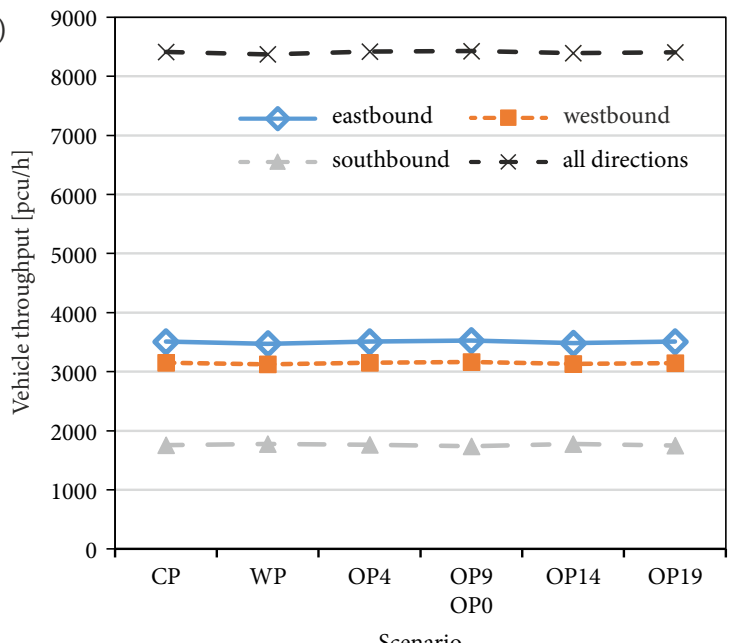

c)

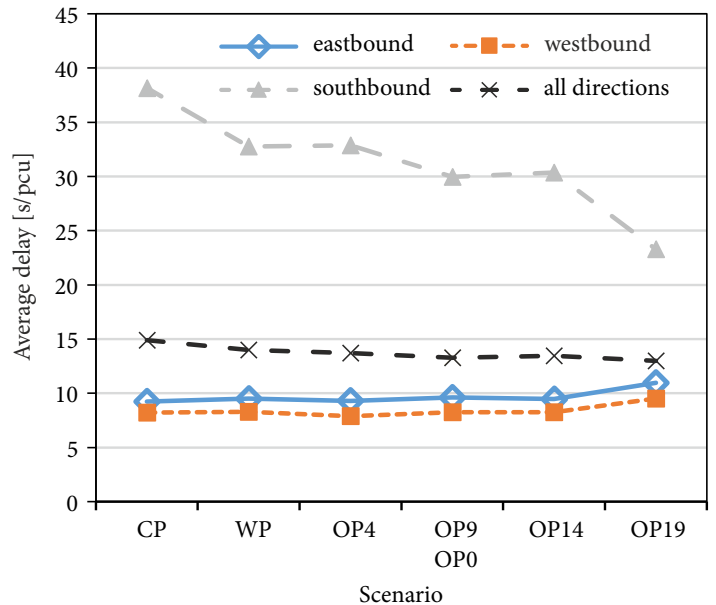

The aforementioned results reveal that the scenarios obtained from the formulated models all perform better than both the CP and the WP; and the model with the minimization of the total delay is relatively easy to generate the optimal scenario of temporal-spacial resources allocation.

\section{Discussions and suggestions}

According to the above analysis, the optimal sequences for these scenarios may be different based on different performance measures. The difference may originate from three aspects:

1) the capacity is theoretical instead of being obtained under real traffic and signal conditions;

2) the settings of travel time sections in VISSIM have an effect on measuring the delay;

3) the random seed has an influence on the performance measures.

On the whole, the optimization models of maximizing the sum of capacity-to-delay ratios, minimizing the total delay and minimizing the weighted sum of delayto-capacity ratios are recommended to apply in practice.

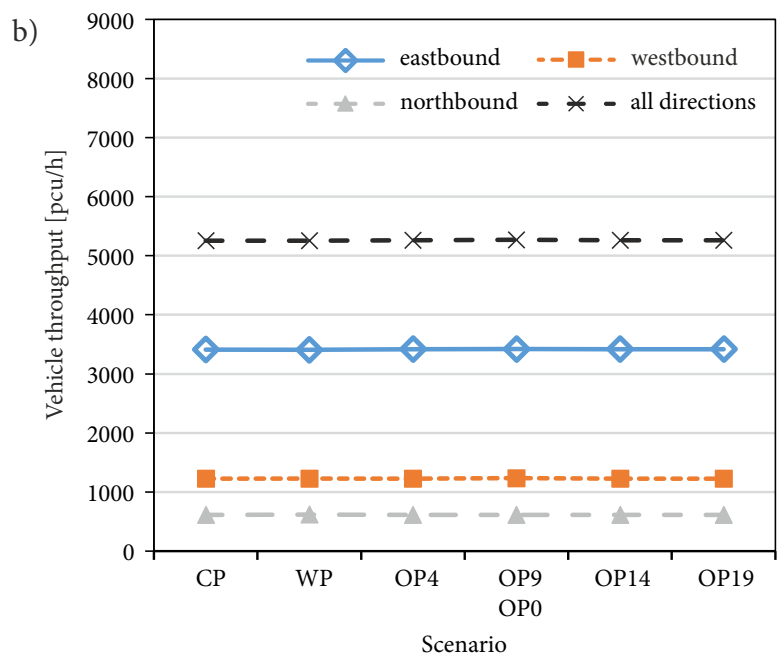

d)

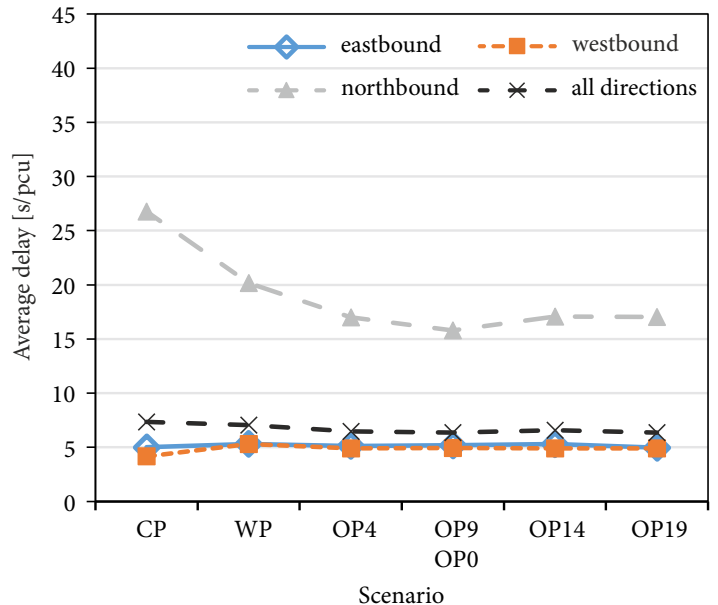

Figure 7. Comparison of vehicle throughputs and average delays under different scenarios: a - vehicle throughputs for intersection A; b - vehicle throughputs for intersection B; c - average delays for intersection A; d - average delays for intersection B 


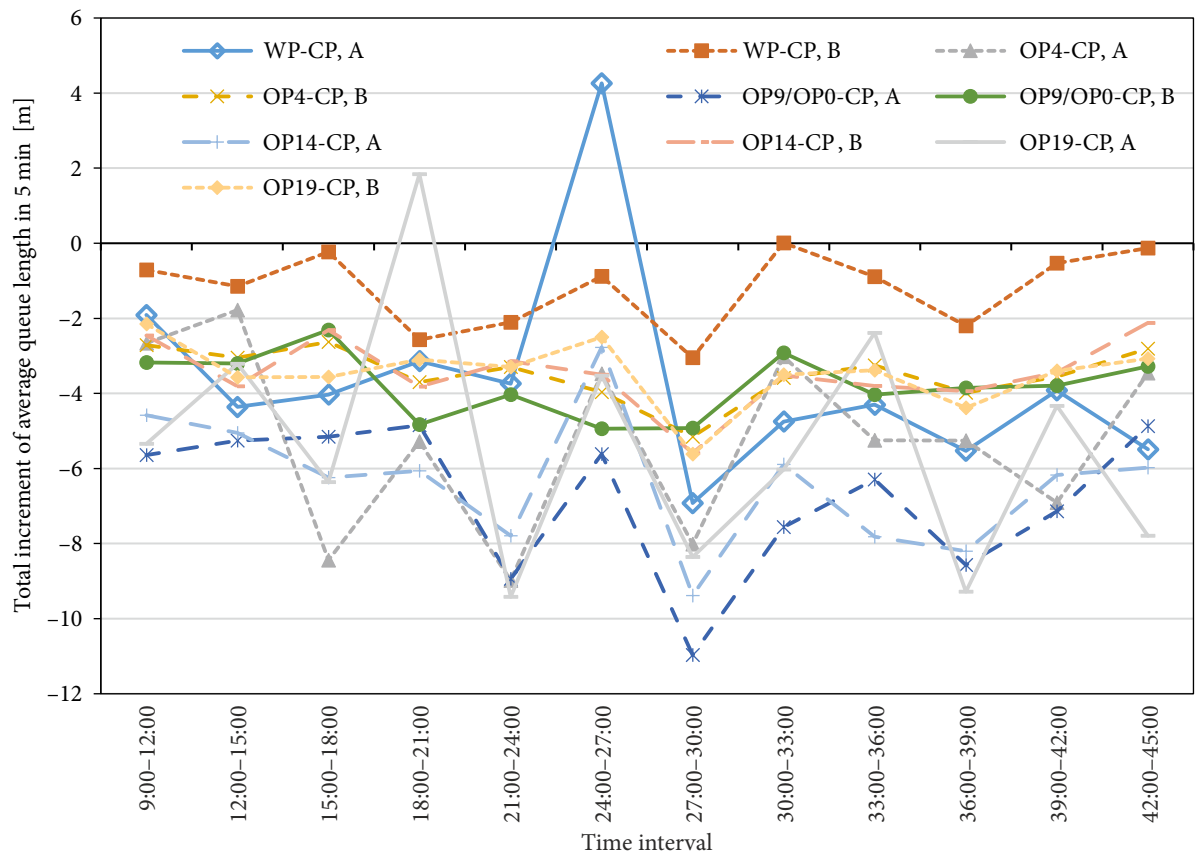

Figure 8 . Total increment of average queue lengths in $5 \mathrm{~min}$

Table 8. Comparison of network performance results

\begin{tabular}{|c|c|c|c|c|c|c|c|}
\hline \multirow{2}{*}{$\begin{array}{l}\text { Statistical } \\
\text { index }\end{array}$} & \multirow[b]{2}{*}{ Scenario } & \multicolumn{6}{|c|}{ Performance measure } \\
\hline & & $\begin{array}{c}\text { Average delay } \\
{[\mathrm{s} / \mathrm{pcu}]}\end{array}$ & $\begin{array}{c}\text { Average } \\
\text { stops }\end{array}$ & $\begin{array}{c}\text { Average speed } \\
{[\mathrm{km} / \mathrm{h}]}\end{array}$ & $\begin{array}{c}\text { Stopped delay } \\
{[\mathrm{s} / \mathrm{pcu}]}\end{array}$ & $\begin{array}{c}\text { Active } \\
\text { vehicles }\end{array}$ & $\begin{array}{l}\text { Arrived } \\
\text { vehicles }\end{array}$ \\
\hline \multirow{6}{*}{ Average } & CP & 22.95 & 0.43 & 42.85 & 9.05 & 451 & 11293 \\
\hline & WP & 21.48 & 0.44 & 43.35 & 8.09 & 399 & 11314 \\
\hline & OP4 & 21.81 & 0.43 & 43.30 & 7.61 & 421 & 11309 \\
\hline & OP9/OP0 & 19.47 & 0.43 & 44.03 & 7.27 & 429 & 11274 \\
\hline & OP14 & 21.68 & 0.44 & 43.30 & 7.41 & 393 & 11313 \\
\hline & OP19 & 20.38 & 0.46 & 43.75 & 6.81 & 437 & 11259 \\
\hline \multirow{6}{*}{ Maximum } & $\mathrm{CP}$ & 34.51 & 0.47 & 44.40 & 9.34 & 515 & 11398 \\
\hline & WP & 31.25 & 0.49 & 44.66 & 8.42 & 467 & 11409 \\
\hline & OP4 & 40.32 & 0.53 & 44.83 & 7.95 & 493 & 11420 \\
\hline & OP9/OP0 & 24.81 & 0.45 & 44.97 & 7.40 & 465 & 11385 \\
\hline & OP14 & 30.15 & 0.51 & 45.10 & 7.77 & 432 & 11406 \\
\hline & OP19 & 31.91 & 0.51 & 45.07 & 7.09 & 518 & 11355 \\
\hline \multirow{6}{*}{ Minimum } & $\mathrm{CP}$ & 18.34 & 0.40 & 39.21 & 8.82 & 403 & 11175 \\
\hline & WP & 17.73 & 0.42 & 40.18 & 7.87 & 357 & 11187 \\
\hline & OP4 & 17.16 & 0.41 & 37.59 & 7.30 & 378 & 11097 \\
\hline & OP9/OP0 & 16.81 & 0.41 & 42.19 & 7.08 & 389 & 11128 \\
\hline & OP14 & 16.46 & 0.41 & 40.52 & 7.14 & 360 & 11207 \\
\hline & OP19 & 16.54 & 0.43 & 39.98 & 6.55 & 394 & 11162 \\
\hline \multirow{6}{*}{$\begin{array}{l}\text { Standard } \\
\text { deviation }\end{array}$} & $\mathrm{CP}$ & 5.16 & 0.02 & 1.66 & 0.17 & 34 & 72 \\
\hline & WP & 4.14 & 0.02 & 1.38 & 0.15 & 30 & 72 \\
\hline & OP4 & 7.05 & 0.04 & 2.22 & 0.19 & 33 & 101 \\
\hline & OP9/OP0 & 2.24 & 0.01 & 0.79 & 0.11 & 27 & 83 \\
\hline & OP14 & 4.59 & 0.03 & 1.55 & 0.19 & 22 & 68 \\
\hline & OP19 & 4.38 & 0.02 & 1.45 & 0.16 & 36 & 67 \\
\hline
\end{tabular}


The weighting factors of the objective functions should be determined by doing the tests although these weighting factors do not have great impacts on the optimization results in the above-mentioned experiments. It is relatively laborious to determine a set of optimum weighting factors, but a better combination of left-turn bay lengths and effective green times can be acquired. As a result, paired intersections with left-turn bays and uncoordinated signals may be in better operation.

The above findings are gotten on a peak-period basis. As is known to all, traffic stream fluctuates within the whole day, and various signal timing scenarios should be used during various time periods. However, intersection layout is generally unchanged during several months or years. The optimal combination of left-turn bay lengths and phase effective green times depends on the traffic de- mands and the optimized values of left-turn bay lengths are required for a specified period. Therefore, the following steps are recommended for use:

1) the optimal combination of intersection temporalspacial resources is obtained using the recommended models;

2) the left-turn bay space is determined by selecting the maximum or the combination of which the sum of the optimized left-turn bay lengths on the road segment between paired intersections is the maximum;

3) the signal timing scheme is determined based on the optimal combination.

The usage of the recommended models is demonstrated for practical applications, as shown in Figure 9.

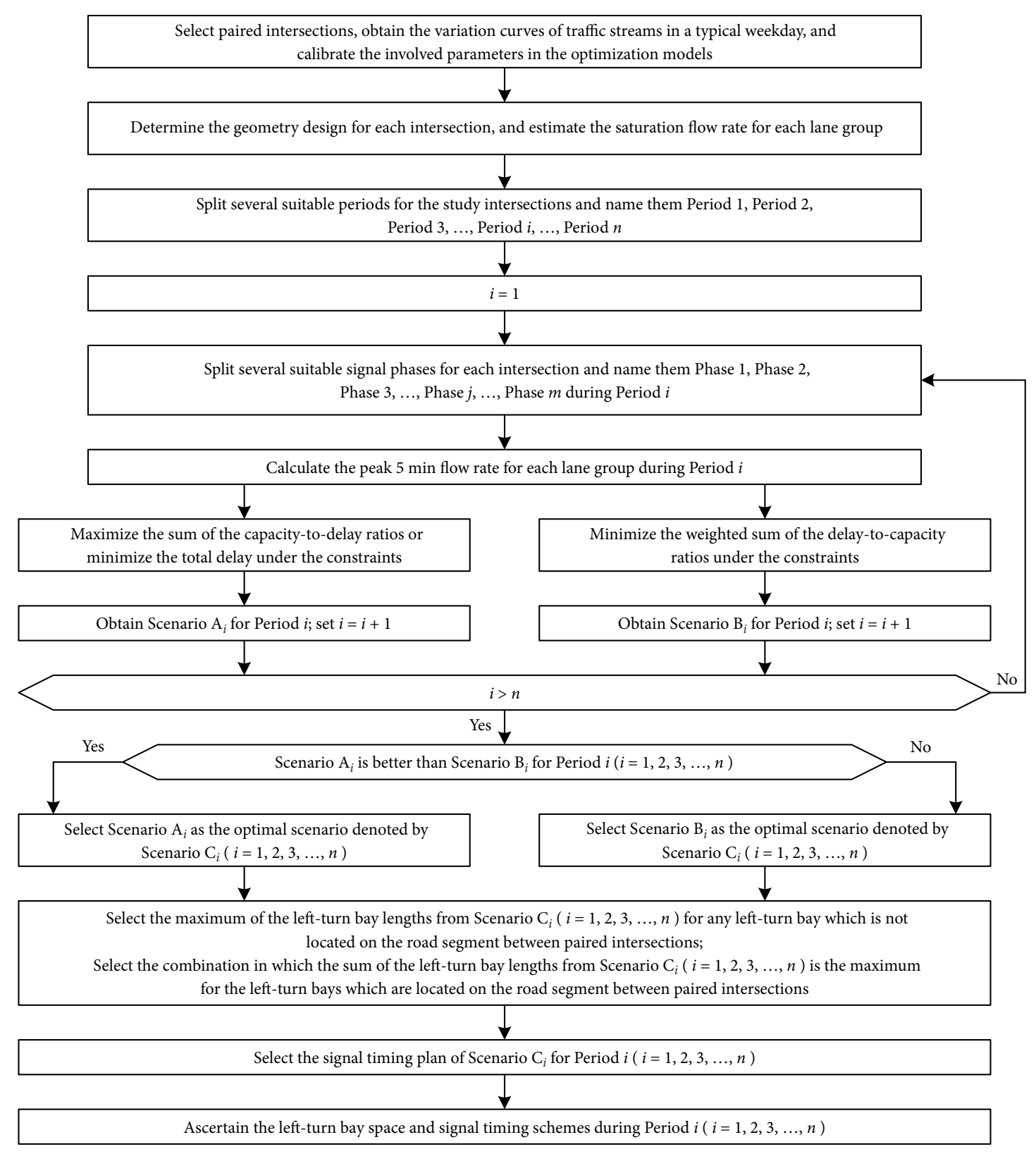

Figure 9. Flow chart of model application 


\section{Conclusions}

At signalized intersections, left-turn bays are often installed and have an important effect on intersection capacity. Four single-objective or multi-objective optimization models are formulated for uncoordinated paired intersections with left-turn bays. To compare different lane arrangement and signal timing scenarios, the total capacity-to-delay ratio is created to measure traffic stream operations. By analysing the sensitivities of the model results to the weighting factors of the objective functions, five preferable optimized scenarios are selected. To validate the influences of the optimized scenarios on traffic stream operations, they are simulated together with the existing (CP) and Webster's (WP) scenarios in VISSIM. The interval estimation and hypothesis testing are used to analyse the sample data from the simulated scenarios. Finally, three concrete models are suggested to use in practice, and the flowchart for applying the recommended models is also proposed.

The contributions of this study are as below. First, the optimization models are developed for uncoordinated paired intersections with left-turn bays. Second, the solutions to these models are proposed and the sensitivities of the optimized outcomes to the weighting factors of the objective functions are testified. Third, the total capacityto-delay ratio is proposed to measure the operations of paired intersections as a whole. Finally, the procedure is presented to apply the optimization models.

When the distance between paired intersections is shorter than the required threshold and one or more movements need to be controlled by coordinated signals, signal coordination should be considered to avoid the queue spillback. In that case, new models need to be formulated.

\section{Acknowledgements}

This research has been funded by the National Natural Science Foundation of China (Grant No 51578111).

The authors also acknowledge the Fundamental Research Funds for the Central Universities of China (Grant No DUT17RW126).

\section{Funding}

This work was supported by the National Natural Science Foundation of China (Grant No 51578111); Fundamental Research Funds for the Central Universities of China (Grant No DUT17RW126).

\section{Author contributions}

Ronghan Yao conceived the study and were responsible for the model formulation and the data analysis.

Ronghan Yao, Wensong Zhang and Meng Long were responsible for the data interpretation.

Ronghan Yao wrote and revised this article.

\section{Disclosure statement}

We have no competing financial, professional, or personal interests from other parties.

\section{References}

Akçelik, R. 1998. Traffic Signals: Capacity and Timing Analysis. Australian Road Research Board (ARRB) Transport Research Ltd, Victoria, Australia. 108 p.

FGSV. 2003. Guidelines for Traffic Signals (RiLSA). Road and Transportation Research Association - Forschungsgesellschaft für Straßen- und Verkehrswesen (FGSV), Cologne, Germany. $182 \mathrm{p}$.

Gan, Y.; Tian, F.; Li, W.; et al. 2005. Operations Research. Tsinghua University Press, Beijing, China. (in Chinese).

Highway Capacity Manual. 2000. Transportation Research Board (TRB), National Research Council. Washington, DC, US. $1134 \mathrm{p}$.

Kikuchi, S.; Kii, M.; Chakroborty, P. 2004. Lengths of double or dual left-turn lanes, Transportation Research Record: Journal of the Transportation Research Board 1881: 72-78. https://doi.org/10.3141/1881-09

Kikuchi, S.; Kronprasert, N. 2010. Determining lengths of leftturn lanes at signalized intersections under different left-turn signal schemes, Transportation Research Record: Journal of the Transportation Research Board 2195: 70-81.

https://doi.org/10.3141/2195-08

Kikuchi, S.; Kronprasert, N.; Kii, M. 2007. Lengths of turn lanes on intersection approaches: three-branch fork lanes-leftturn, through, and right-turn lanes, Transportation Research Record: Journal of the Transportation Research Board 2023: 92-101. https://doi.org/10.3141/2023-10

Lee, J.-J.; Rouphail, N. M.; Hummer, J. E. 2005. Models for lane utilization prediction for lane drop intersections, Transportation Research Record: Journal of the Transportation Research Board 1912: 47-56. https://doi.org/10.3141/1912-06

Liu, M.; Xu, L.; Shen, L. X.; Jin, S. 2019. Modeling capacity at signalized intersections with a left-turn storage bay considering signal timing plan, Journal of Transportation Engineering, Part A: Systems 145(2): 04018084.

https://doi.org/10.1061/JTEPBS.0000210

Osei-Asamoah, A.; Kulshrestha, A.; Washburn, S. S.; Yin, Y. 2010. Impact of left-turn spillover on through movement discharge at signalized intersections, Transportation Research Record: Journal of the Transportation Research Board 2173: 80-88. https://doi.org/10.3141/2173-10

PTV. 2012. PTV VISSIM 5.40 User Manual. Planung Transport Verkehr (PTV) AG, Karlsruhe, Germany.

PTV. 2014. PTV VISSIM 6.00 User Manual. Planung Transport Verkehr (PTV) AG, Karlsruhe, Germany.

Qi, Y.; Guo, L.; Yu, L.; Teng, H. 2012. Estimation of design lengths of left-turn lanes, Journal of Transportation Engineering 138(3): 274-283. https://doi.org/10.1061/(ASCE)TE.1943-5436.0000299

Qi, Y.; Yu, L.; Azimi, M.; Guo, L. 2007. Determination of storage lengths of left-turn lanes at signalized intersections, Transportation Research Record: Journal of the Transportation Research Board 2023: 102-111. https://doi.org/10.3141/2023-11

Quan, Y. S. 1989. Urban Traffic Control. China Communications Press Ltd. (in Chinese).

Sun, J. 2014. Guidelines for Microscopic Traffic Simulation Analysis. Tongji University Press, Shanghai, China. (in Chinese). 
Tageldin, A.; Sayed, T.; Ismail, K. 2018. Evaluating the safety and operational impacts of left-turn bay extension at signalized intersections using automated video analysis, Accident Analysis \& Prevention 120: 13-27.

https://doi.org/10.1016/j.aap.2018.07.029

Tian, Z. Z.; Wu, N. 2006. Probabilistic model for signalized intersection capacity with a short right-turn lane, Journal of Transportation Engineering 132(3): 205-212.

https://doi.org/10.1061/(ASCE)0733-947X(2006)132:3(205)

Wu, B.; Li, Y. 2015. Traffic Management and Control. 5th Edition. China Communications Press Ltd. (in Chinese).

$\mathrm{Wu}, \mathrm{N} .2007$. Total approach capacity at signalized intersections with shared and short lanes: generalized model based on a simulation study, Transportation Research Record: Journal of the Transportation Research Board 2027: 19-26.

https://doi.org/10.3141/2027-03

Yang, J.; Zhou, H. 2011. Integrating left-turn lane geometric design with signal timing, Journal of Transportation Engineering 137(11): 767-774.

https://doi.org/10.1061/(ASCE)TE.1943-5436.0000282

Yao, R. 2013a. Sensitivity analysis of optimization models for isolated intersections with short left-turn lanes on approaches, Journal of Advanced Transportation 47(1): 28-42. https://doi.org/10.1002/atr.1185

Yao, R. 2013b. Sensitivity analysis of optimization models for two adjacent intersections with correlated short left-turn lanes, Transport 28(3): 256-269.

https://doi.org/10.3846/16484142.2013.829781

Yao, R. 2016. Settings of short left-turn lane and signal phase sequence for isolated signalized intersections, Transport 31(4): 416-426. https://doi.org/10.3846/16484142.2014.915427

Yao, R.; Wang, J.; Jia, J. 2012. Synergistic optimization of short lane length and signal timing parameters for two adjacent intersections, Journal of Dalian University of Technology 52(4): 546-552. (in Chinese).

Yao, R.; Wang, J.; Wang, T.; Zhu, C. 2011. Synergistic optimization model of length and signal timing parameters of left-turn short lane, Communications Standardization (9): 167-172. (in Chinese).

Yin, K.; Zhang, Y.; Wang, B. X. 2010. Analytical models for protected plus permitted left-turn capacity at signalized intersection with heavy traffic, Transportation Research Record: Journal of the Transportation Research Board 2192: 177-184. https://doi.org/10.3141/2192-17

Yin, K.; Zhang, Y.; Wang, B. X. 2011. Modeling delay during heavy traffic for signalized intersections with short left-turn bays, Transportation Research Record: Journal of the Transportation Research Board 2257: 103-110. https://doi.org/10.3141/2257-12

Zhang, K. Y.; Gao, W. S. 1997. Probability Theory and Mathematical Statistics. Northeast Normal University Press, Changchun, China. (in Chinese).

Zhang, Y.; Tong, J. 2008. Modeling left-turn blockage and capacity at signalized intersection with short left-turn bay, Transportation Research Record: Journal of the Transportation Research Board 2071: 71-76. https://doi.org/10.3141/2071-09

Zheng, L.; Sayed, T.; Tageldin, A. 2018. Before-after safety analysis using extreme value theory: a case of left-turn bay extension, Accident Analysis \& Prevention 121: 258-267.

https://doi.org/10.1016/j.aap.2018.09.023 